\title{
CHARACTERISTIC SUBGROUPS OF LATTICE-ORDERED GROUPS $\left({ }^{1}\right)$
}

\author{
BY \\ RICHARD D. BYRD, PAUL CONRAD AND JUSTIN T. LLOYD
}

\begin{abstract}
Characteristic subgroups of an $l$-group are those convex $l$-subgroups that are fixed by each $l$-automorphism. Certain sublattices of the lattice of all convex $l$ subgroups determine characteristic subgroups which we call socles. Various socles of an $l$-group are constructed and this construction leads to some structure theorems. The concept of a shifting subgroup is introduced and yields results relating the structure of an $l$-group to that of the lattice of characteristic subgroups. Interesting results are obtained when the $l$-group is characteristically simple. We investigate the characteristic subgroups of the vector lattice of real-valued functions on a root system and determine those vector lattices in which every $l$-ideal is characteristic. The automorphism group of the vector lattice of all continuous real-valued functions (almost finite real-valued functions) on a topological space (a Stone space) is shown to be a splitting extension of the polar preserving automorphisms by the ring automorphisms. This result allows us to construct characteristically simple vector lattices. We show that self-injective vector lattices exist and that an archimedean self-injective vector lattice is characteristically simple. It is proven that each $l$-group can be embedded as an $l$-subgroup of an algebraically simple $l$-group. In addition, we prove that each representable (abelian) $l$-group can be embedded as an $l$-subgroup of a characteristically simple representable (abelian) $l$-group.
\end{abstract}

1. Introduction. A convex $l$-subgroup $C$ of an $l$-group $G$ is called characteristic if $C \tau=C$ for each $l$-automorphism $\tau$ of $G$. In this paper we investigate the characteristic subgroups of $G$ and in the process construct various characteristically simple l-groups.

Let $\mathscr{C}(G), \mathscr{L}(G)$ and $\mathscr{K}(G)$ be the lattices of all convex $l$-subgroups, $l$-ideals, and characteristic subgroups of $G$, respectively. Each lattice determines a socle for $G$ namely the cardinal sum of all the atoms. Each of these socles is a characteristic subgroup of $G$. Here the fact that complements of cardinal summands in an $l$-group are unique enables one to obtain much more theory than one gets for groups or

Received by the editors February 24, 1970.

AMS 1969 subject classifications. Primary 0675, 0685.

Key words and phrases. Socles of an $l$-group, $l$-automorphism, characteristic subgroup, characteristically simple l-group, polar, Boolean algebra, completely reducible l-group, shifting subgroup, $s$-simple subgroup, completely $s$-reducible l-group, lex-subgroup, prime subgroup, closed subgroup, cardinally indecomposable $l$-group, lex-extension, basic element, basis, principal polar, lex-kernel, regular subgroup, special element, finite valued $l$-group, root system, root, vector lattice, essential extension, archimedean extension, completely regular space, real compact space, splitting extension, self-injective l-group, large subgroup, hyperarchimedean $l$-group, radical, ideal radical, distributive radical, singular element.

(1) This work was supported in part by National Science Foundation grants.

Copyright (C) 1971, American Mathematical Society 
abelian groups. In $\$ 2$ we give a construction that produces all of the above socles and more. This leads to various structure theorems for $G$. For example, for an $l$-group $G$ (representable $l$-group $G$ ) the following are equivalent:

(a) $G$ is characteristically simple and contains a minimal $l$-ideal.

(b) $G$ is a cardinal sum of $l$-isomorphic simple $l$-groups (simple $o$-groups).

(c) $G$ is characteristically simple and completely reducible.

An element $C \in \mathscr{C}(G)$ is called shifting if $C \tau=C$ or $C \tau \cap C=0$ for each $l$ automorphism $\tau$ of $G$. In particular, each characteristic subgroup is shifting and, if $C \in \mathscr{C}(G)$ is minimal with respect to being a convex $l$-subgroup, $l$-ideal, polar, lex-subgroup, or a cardinal summand, then $C$ is an s-subgroup. This concept allows us to identify characteristically simple $l$-groups. In fact, a characteristically simple $l$-group $G$ is a cardinal sum of $l$-isomorphic $s$-simple $s$-subgroups. Moreover, these summands together with $G$ and 0 are all the $s$-subgroups of $G$. Thus if $G$ is characteristically simple, then each s-subgroup is a cardinal summand, and we say that $G$ is completely s-reducible. In Theorem 3.23 we derive eight conditions each of which is equivalent to $G$ being completely $s$-reducible-for example, $\mathscr{K}(G)$ is a complete Boolean algebra; each characteristic subgroup is a cardinal summand; $G$ is a cardinal sum of $s$-simple $s$-subgroups.

In $\S 4$ we investigate characteristically simple $l$-groups. In order to identify such groups one needs to know something about the group $A(G)$ of all $l$-automorphisms of $G$. In $\S 6$ we investigate $A(G)$ when $G=C(X)$, the vector lattice of all continuous real-valued functions on a topological space $X$ or $G=D(X)$, the vector lattice of all almost finite real-valued functions on a Stone space $X$. In either case $A(G)$ is a splitting extension of the polar preserving automorphisms of $G$ by the ring automorphisms of $G$ and this result allows us to construct characteristically simple vector lattices.

One of the main results about abelian $l$-groups asserts that each such group can be embedded in a vector lattice $V=V\left(\Lambda, R_{\lambda}\right)$ of real-valued functions. In $\S 5$ we investigate the characteristic subgroups of $V$. We also determine those $V$ 's for which every $l$-ideal is characteristic.

It is known that the category of vector lattices does not admit any injectives. In $\$ 7$ we show that self-injectives do exist and that an archimedean self-injective vector lattice is characteristically simple. We also take a look at the relationship between hyper-archimedean and self-injective vector lattices.

In $\S 8$ we prove that each $l$-group $G$ can be embedded in an algebraically simple $l$-group and hence in a characteristically simple $l$-group. Moreover we show that each representable (abelian) $l$-group can be embedded in a representable (abelian) characteristically simple $l$-group. The last section consists of examples that show the extent as well as the limitations of our theory.

2. The socles of an l-group. We will denote by $A(G)$ the group of $l$-automorphisms of the $l$-group $G$. A convex $l$-subgroup $C$ of $G$ is said to be characteristic 
if $C \tau=C$ for each $\tau \in A(G) . G$ is characteristically simple if the only characteristic subgroups of $G$ are $G$ and 0 , and $G$ is simple if $G$ and 0 are the only $l$-ideals of $G$. If $\left\{G_{\lambda} \mid \lambda \in \Lambda\right\}$ is a collection of $l$-groups, then $\sum G_{\lambda}\left(\Pi G_{\lambda}\right)$ denotes the cardinal sum (cardinal product) of the l-groups $G_{\lambda}$. In case $\Lambda=\{1,2, \ldots, n\}$, we will write $G_{1} \boxplus G_{2} \boxplus \cdots \boxplus G_{n}$ instead of $\sum G_{\lambda}$. If $T$ is a subset of $G$, then [T] denotes the subgroup of $G$ that is generated by $T$ and $T^{\prime}$ denotes the polar of $T$, that is $T^{\prime}=$ $\{x \in G|| x|\wedge| t \mid=0$ for all $t \in T\} . G$ is said to be representable if there is an $l-$ isomorphism of $G$ into a cardinal product of totally ordered groups. It is well known that an $l$-group $G$ is representable if and only if each (principal) polar of $G$ is an $l$-ideal. Throughout this paper $\mathscr{C}(G)(\mathscr{L}(G), \mathscr{K}(G))$ will denote the lattice of all convex $l$-subgroups ( $l$-ideals, characteristic subgroups) of $G$.

Let $\mathscr{S}$ be a property possessed by certain convex $l$-subgroups of $G$; for example, the property of being normal, characteristic, a polar, etc. Let $\mathscr{S}(G)$ denote the collection of all convex l-subgroups with property $\mathscr{S}$. An element of $\mathscr{S}(G)$ will be called an $\mathscr{S}$-subgroup. If $\mathscr{S}(G)$ consists only of $G$ and 0 , then we shall say that $G$ is $\mathscr{S}$-simple. For the remainder of this section we suppose that $\mathscr{S}(G)$ is a complete sublattice of $\mathscr{C}(G)$ containing $G$ and 0 and such that

(i) If $C \in \mathscr{S}(G)$, then $C^{\prime} \in \mathscr{S}(G)$.

(ii) If $G=A \boxplus B$ and $C \in \mathscr{S}(G)$, then $C \cap A \in \mathscr{S}(A)$.

(iii) If $G=A \boxplus B$ and $A$ is an atom in $\mathscr{S}(G)$, then $A$ is $\mathscr{S}$-simple.

Let $\left\{C_{i} \mid i \in I\right\}$ be the set of all atoms in $\mathscr{S}(G)$. Then $C_{i} \cap C_{j}=0$ for $i \neq j$, and hence $\bigvee C_{i}=\sum C_{i}$, and, of course, $\bigvee C_{i} \in \mathscr{S}(G)$. We shall call $\bigvee C_{i}$ the $\mathscr{S}$-socle of $G$.

Proposition 2.1. $\mathscr{C}(G), \mathscr{L}(G)$, and $\mathscr{K}(G)$ are complete sublattices of $\mathscr{C}(G)$ containing $G$ and 0 and satisfying conditions (i), (ii), and (iii) above. Moreover

(a) Each atom in $\mathscr{C}(G)$ is an archimedean o-group and hence o-isomorphic to a subgroup of the reals.

(b) Each atom in $\mathscr{K}(G)$ is a characteristically simple l-group.

(c) If $A$ is an atom in $\mathscr{L}(G)$ and a cardinal summand of $G$, then $A$ is a simple lgroup. If, in addition, $G$ is representable, then each atom in $\mathscr{L}(G)$ is an o-group.

Proof. Clearly $\mathscr{C}(G)$ and $\mathscr{L}(G)$ satisfy (i), (ii), and (iii). If $C \in \mathscr{K}(G)$ and $\tau \in A(G)$, then $C^{\prime} \tau=(C \tau)^{\prime}=C^{\prime}$ and hence (i) is satisfied. Suppose that $G=A \boxplus B$, let $C \in \mathscr{K}(G)$, and let $\alpha$ be an $l$-automorphism of $A$. Let $\tau$ be the extension of $\alpha$ to $G$ such that $\tau$ induces the identity on $B$. Then $(A \cap C) \alpha=(A \cap C) \tau=A \tau \cap C \tau=$ $A \cap C$. Thus $A \cap C \in \mathscr{K}(A)$ and hence (ii) is satisfied. Since each atom in $\mathscr{K}(G)$ is characteristically simple, (iii) is satisfied.

(a), (b), and the first part of (c) are clear. Suppose that $G$ is representable and let $A$ be an atom in $\mathscr{L}(G)$. If $0<a \in A$, then $a^{\prime}$ is an $l$-ideal and $a \notin a^{\prime}$. Thus $A \cap a^{\prime}=0$. It follows that $A$ contains no pair of disjoint elements and is therefore an $o$-group.

THEOREM 2.2. For an l-group $G$, the following are equivalent:

(a) Each $C \in \mathscr{S}(G)$ is a cardinal summand.

(b) $G$ is the $\mathscr{S}$-socle of $G$. 
(c) $G$ is a cardinal sum of $\mathscr{S}$-simple l-groups.

(d) $G$ is a join of atoms from $\mathscr{S}(G)$.

(e) $\mathscr{S}(G)$ is a complete, atomic, Boolean algebra.

(f) $\mathscr{S}(G)$ is a Boolean algebra.

Proof. (a) implies (b). Suppose (by way of contradiction) that $G \neq S$ where $S$ denotes the $\mathscr{S}$-socle of $G$. Let $a \in G \backslash S$ and let $T$ be maximal in $\mathscr{S}(G)$ with respect to $S \subseteq T$ and $a \notin T$; and let $R$ be the intersection of all members of $\mathscr{S}(G)$ that properly contain $T$. Then $R \in \mathscr{S}(G)$. Now $G=T \boxplus K$ and so $R=(R \cap T) \boxplus(R \cap K)=$ $T \boxplus(R \cap K)$. Clearly $(R \cap K)$ must be an atom in $\mathscr{S}(G)$, but this implies that $R \cap K \subseteq S \subseteq T$, a contradiction. Thus $S=G$.

(b) implies (c). This is an immediate consequence of (iii).

(c) implies (d). Suppose that $G=\sum G_{\lambda}(\lambda \in \Lambda)$, where each $G_{\lambda}$ is an $\mathscr{S}_{\text {-simple }}$ l-group. If $T \in \mathscr{S}(G)$, then $T=\sum\left(G_{\lambda} \cap T\right)(\lambda \in \Lambda)$. By (ii), $G_{\lambda} \cap T \in \mathscr{S}\left(G_{\lambda}\right)$ and, since $G_{\lambda}$ is $\mathscr{S}$-simple, we have for each $\lambda \in \Lambda$ that $G_{\lambda} \cap T=0$ or $G_{\lambda} \cap T=G_{\lambda}$. Let $\lambda_{0} \in \Lambda$ and let $T_{\lambda_{0}}$ be the $\mathscr{S}$-subgroup of $G$ generated by $G_{\lambda_{0}}$. Then $T_{\lambda_{0}}=\sum G_{\delta}$ $(\delta \in \Delta \subseteq \Lambda)$. We prove that $T_{\lambda_{0}}$ is an atom in $\mathscr{S}(G)$. Suppose (by way of contradiction) that $C \in \mathscr{S}(G)$ and $0 \neq C \subset T_{\lambda_{0}}$. Then $C=\sum G_{\gamma}(\gamma \in \Gamma, \Gamma \subseteq \Delta)$ and $\lambda_{0} \notin \Gamma$. By (i) $C^{\prime}=\sum G_{\lambda}(\lambda \in \Lambda \mid \Gamma) \in \mathscr{S}(G)$, and so $T_{\lambda_{0}} \subseteq C^{\prime}$. From this we conclude that $C \subset C^{\prime}$, which is a contradiction since $C \neq 0$. Thus $T_{\lambda_{0}}$ is an atom in $\mathscr{S}(G)$. It follows that $G$ is the join of atoms in $\mathscr{S}(G)$.

(d) implies (e). If $S_{i}$ and $S_{j}$ are distinct atoms in $\mathscr{S}(G)$, then $S_{i} \cap S_{j}=0$ and so $G=\sum S_{j}(j \in J)$, where $\left\{S_{j} \mid j \in J\right\}$ is the set of all atoms in $\mathscr{S}(G)$. If $T \in \mathscr{S}(G)$, then $T=\sum\left(S_{j} \cap T\right)(j \in J)$ and $S_{j} \cap T=0$ or $S_{j} \cap T=S_{j}$. Thus $\mathscr{S}(G)$ is isomorphic to the set of all subsets of $J$.

The implications (e) implies (f) and (f) implies (a) are trivial.

Note that we do not assume that the summands of part (c) of Theorem 2.2 are elements of $\mathscr{S}(G)$ (see Example 9.2). We leave it to the reader to formulate the special cases of the theorem when $\mathscr{S}(G)=\mathscr{C}(G), \mathscr{L}(G)$, or $\mathscr{K}(G)$. Note that the atoms in each of these three cases are described in Proposition 2.1.

An $l$-group $G$ is said to be completely reducible if each $l$-ideal of $G$ is a cardinal summand. Thus we have shown that $G$ is completely reducible if and only if $G$ is a cardinal sum of simple $l$-groups. If this is the case, then $G$ is abelian if and only if $\mathscr{C}(G)=\mathscr{L}(G)$. An abelian l-group is completely reducible if and only if it is a cardinal sum of subgroups of the reals. A representable l-group is completely reducible if and only if it is a cardinal sum of simple $o$-groups. These facts are, of course, immediate consequences of Proposition 2.1 and Theorem 2.2.

COROLlaRY 2.3. For an l-group $G$, the following are equivalent:

(a) $G$ is characteristically simple and $\mathscr{C}(G)$ contains an atom.

(b) $G$ is a cardinal sum of o-isomorphic archimedean o-groups.

(c) $G$ is characteristically simple and each convex l-subgroup of $G$ is a cardinal summand. 
Proof. (a) implies (b). Let $\mathscr{S}(G)=\mathscr{C}(G)$. If $S$ is the $\mathscr{S}$-socle of $G$, then $S \neq 0$ and is a characteristic subgroup of $G$. Hence $G=S=\sum S_{i}$, where $\left\{S_{i} \mid i \in I\right\}$ is the set of atoms of $\mathscr{C}(G)$. By Proposition 2.1, each $S_{i}$ is an archimedean $o$-group and, since $G$ is characteristically simple, $S_{i}$ is $o$-isomorphic to $S_{j}$ for $i, j \in I$.

The implications (b) implies (c) and (c) implies (a) follow from the theorem.

COROLlary 2.4. For an l-group $G$ (representable l-group $G$ ) the following are equivalent:

(a) $G$ is characteristically simple and contains a minimal l-ideal.

(b) $G$ is a cardinal sum of l-isomorphic simple l-groups (simple o-groups).

(c) $G$ is characteristically simple and completely reducible.

Proof. Let $\mathscr{S}(G)=\mathscr{L}(G)$ and proceed as in Corollary 2.3.

COROLlARY 2.5. For an l-group $G$, the following are equivalent:

(a) $G$ is characteristically simple and contains an atom.

(b) $G$ is a cardinal sum of cyclic o-groups.

Proof. (a) implies (b). If $0<x$ is an atom in $G$, then [x] is an atom in $\mathscr{C}(G)$ and hence, by Corollary 2.3, $G=\sum S_{i}$, where the $S_{i}$ 's are $o$-isomorphic archimedean $o$-groups, and clearly one of them is $[x]$.

The implication (b) implies (a) is trivial.

THEOREM 2.6. If there exists a set of maximal $\mathscr{S}$-subgroups of $G$ which are polars and whose intersection is zero, then there exists an l-isomorphism $\tau$ of $G$ such that

$$
\sum G_{\lambda} \subseteq G \tau \subseteq \prod G_{\lambda}
$$

where each $G_{\lambda}$ is an atom in $\mathscr{S}(G)$. In particular, $\sum G_{\lambda}$ is the $\mathscr{S}$-socle of $G$.

Proof. Suppose that $M=M^{\prime \prime}$ is a maximal $\mathscr{S}$-subgroup. Then $M^{\prime} \neq 0$ and $M \subset M \boxplus M^{\prime} \in \mathscr{S}(G)$. Since $M$ is maximal in $\mathscr{S}(G)$, we conclude that $G=M \boxplus M^{\prime}$. Now let $\left\{G^{\lambda} \mid \lambda \in \Lambda\right.$ be a collection of maximal $\mathscr{S}$-subgroups such that $G^{\lambda}=\left(G^{\lambda}\right)^{\prime \prime}$ and $\cap G^{\lambda}=0$. For each $\lambda \in \Lambda, G=G_{\lambda} \boxplus G^{\lambda}$ where clearly $G_{\lambda}$ is an atom in $\mathscr{S}(G)$. Each $g \in G$ has a unique representation of the form $g=g_{\lambda}+g^{\lambda}$, where $g_{\lambda} \in G_{\lambda}$ and $g^{\lambda} \in G^{\lambda}$. Then the mapping $\tau$ given by $g \rightarrow g \tau=\left(\ldots, g_{\lambda}, \ldots\right)$ is an $l$-isomorphism of $G$ into $\prod G_{\lambda}$. If $\alpha$ and $\beta$ are distinct members of $\Lambda$, then $G_{\alpha} \cap G_{\beta}=0$ since $G_{\alpha}$ and $G_{\beta}$ are distinct atoms in $\mathscr{S}(G)$. Let $g \in G_{\alpha}$ and $\lambda \in \Lambda(\lambda \neq \alpha)$. Then $G_{\alpha} \cap G_{\lambda}=0$ implies that $G_{\alpha} \subseteq G_{\lambda}^{\prime}=G^{\lambda}$. Thus $g=g_{\lambda}+g^{\lambda}=0+g$. Therefore $\sum G_{\lambda} \subseteq G \tau$.

We give a method for producing other $\mathscr{S}$-socles for an $l$-group $G$. Let $S$ be a function that assigns to each subgroup $C$ of $G$ a subgroup $S(C)$ of $A(C)$ such that

(a) If $G=C \boxplus D$ and $\alpha \in S(C)$, then $\alpha$ can be extended to an element of $S(G)$ that is the identity on $D$.

(b) If $G=C \boxplus D$ and $C \tau=C$ for some $\tau \in S(G)$, then $\tau \mid C \in S(C)$.

Proposition 2.7. Let $S$ be defined as above and let $\mathscr{S}(G)=\{C \in \mathscr{C}(G) \mid C \tau=C$ for each $\tau \in S(G)\}$. Then $\mathscr{S}(G)$ is a complete sublattice of $\mathscr{C}(G)$ that contains $G$ and 0 
and satisfies (i), (ii), and (iii). Moreover, if $S_{1}$ and $S_{2}$ satisfy (a) and (b) above and if $\mathscr{S}_{i}(G)=\left\{C \in \mathscr{C}(G) \mid C \tau=C\right.$ for each $\left.\tau \in S_{i}(G)\right\}(i=1,2)$, then $\mathscr{S}_{1}(G) \cap \mathscr{S}_{2}(G)$ satisfies the conclusions of the proposition.

Proof. Note that $\mathscr{K}(G) \subseteq \mathscr{S}(G)$, hence $G$ and 0 are elements of $\mathscr{S}(G)$. If $\left\{C_{i} \mid i \in I\right\} \subseteq \mathscr{S}(G)$ and $\tau \in S(G)$, then $\left(\bigvee C_{i}\right) \tau=\bigvee\left(C_{i} \tau\right)=\bigvee C_{i}$ and $\left(\bigwedge C_{i}\right) \tau=$ $\bigwedge\left(C_{i} \tau\right)=\bigwedge C_{i}$. Therefore $\mathscr{S}(G)$ is a complete sublattice of $\mathscr{C}(G)$.

If $C \in \mathscr{S}(G)$, then $C \tau=C$ for all $\tau \in S(G)$ and so $C^{\prime} \tau=(C \tau)^{\prime}=C^{\prime}$ for all $\tau \in S(G)$ and hence $C^{\prime} \in \mathscr{S}(G)$. Let $G=A \boxplus B, C \in \mathscr{S}(G)$, and $\alpha \in S(A)$. Then, by (a), $\alpha$ can be extended to an element $\tau \in S(G)$ that is the identity on $B$. Thus $(C \cap A) \alpha=$ $(C \cap A) \tau=C \tau \cap A \tau=C \cap A$. Therefore $C \cap A \in \mathscr{S}(A)$. Suppose that $G=A \boxplus B$ and that $A$ is an atom in $\mathscr{S}(G)$. Let $0 \neq D \in \mathscr{S}(A)$ and $\tau \in S(G)$. Then $\alpha=\tau \mid A \in S(A)$ and so $D \tau=(D \cap A) \tau=(D \cap A) \alpha=D \alpha \cap A \alpha=D \cap A=D$. Thus $D \in \mathscr{S}(G)$ and it follows that $D=A$. Therefore $A$ is $\mathscr{S}$-simple.

Clearly $\mathscr{S}_{1}(G) \cap \mathscr{S}_{2}(G)$ is a complete sublattice of $\mathscr{C}(G)$ containing 0 and $G$ and satisfying (i), (ii), and (iii).

Note that if $S(G)=A(G)$, then $\mathscr{S}(G)=\mathscr{K}(G)$; if $S(G)=I(G)$, the group of inner automorphisms of $G$, then $\mathscr{S}(G)=\mathscr{L}(G)$; and if $S(G)$ consists only of the identity of $A(G)$, then $\mathscr{S}(G)=\mathscr{C}(G)$. Another example of $S(G)$ that satisfies (a) and (b) is

$$
P(G)=\{\alpha \in A(G) \mid x \wedge y=0 \text { implies } x \wedge y \alpha=0 \text { for all } x, y \in G\} .
$$

This is the group of polar preserving automorphisms of $G$ which we will investigate at some length in $\$ 6$.

In the following proposition, we use the notation established above.

Proposition 2.8. If $S(G)=P(G)$, then the following are equivalent:

(1) $G$ is the $\mathscr{S}$-socle of $G$.

(2) $G$ is the cardinal sum of characteristically simple o-groups.

(3) $\mathscr{S}(G)$ consists of polars.

(4) $\mathscr{S}(G)$ is a complete, atomic, Boolean algebra.

Proof. (1) implies (2). $G=\sum G_{i}(i \in I)$ where each $G_{i}$ is an atom in $\mathscr{S}(G)$. Thus each atom is a polar and hence a minimal polar, but a minimal polar in an l-group is an $o$-group. Thus each $G_{i}$ is an $o$-group. Each characteristic subgroup of $G_{i}$ belongs to $\mathscr{S}(G)$ and so $G_{i}$ is characteristically simple.

(2) implies (3). If $H$ is an $o$-group, then $P(H)=A(H)$. Thus a characteristically simple $o$-group is $\mathscr{S}$-simple. By Theorem 2.2, each $C$ in $\mathscr{S}(G)$ is a cardinal summand and hence a polar.

(3) implies (4). $\mathscr{S}(G)$ is the set of all polars and also a complete sublattice of $\mathscr{C}(G)$. But the collection of polars is a Boolean algebra, and hence, by Theorem 2.2, $\mathscr{S}(G)$ is a complete, atomic, Boolean algebra.

(4) implies (1). This is immediate from Theorem 2.2.

Note that if (1) through (4) hold, then each polar of $G$ is a cardinal summand and so the polars form a sublattice of $\mathscr{L}(G)$. The following example shows that this 
condition does not, in general, imply that the polars form a complete sublattice of $\mathscr{L}(G)$. Let $G=\prod G_{\lambda}(\lambda \in \Lambda)$, where $\Lambda$ is an infinite set and each $G_{\lambda}$ is a nonzero $o$-group. For each $\lambda \in \Lambda$, let

$$
C_{\lambda}=\left\{g \in G \mid g_{\gamma}=0 \text { for } \gamma \neq \lambda\right\} .
$$

Then $\bigvee C_{\lambda}=\sum G_{\lambda}$ and is not a polar, and so the polars do not form a complete sublattice of $\mathscr{L}(G)$. Consider a polar $T$ and let

$$
\Delta=\left\{\lambda \in \Lambda \mid \text { the projection of } T \text { into } G_{\lambda} \text { is not zero }\right\} \text {. }
$$

Then $T \subseteq\left\{g \in G \mid g_{\lambda}=0\right.$ for all $\left.\lambda \in \Lambda \mid \Delta\right\}=\left(\bigvee C_{\delta}\right)^{\prime \prime}(\delta \in \Delta)$. If $0<t \in T$ with $t_{\delta}>0$, then $\delta \in \Delta$, and since $T$ is convex, $g=\left(\ldots, 0, t_{\delta}, 0, \ldots\right)$ belongs to $T$. Thus $C_{\delta}=g^{\prime \prime} \subseteq T$, hence $T \supseteq \bigvee C_{\delta}$ and so $T=T^{\prime \prime} \supseteq\left(\bigvee C_{\delta}\right)^{\prime \prime}$. Therefore $T$ is a cardinal summand of $G$.

Proposition 2.9. (1) If $S(G)$ is a normal subgroup of $A(G)$, then each l-automorphism of $G$ permutes the elements in $\mathscr{S}(G)$, and, in particular, the $\mathscr{S}$-socle is characteristic.

(2) If each l-automorphism of $G$ permutes the elements in $\mathscr{S}(G)$, then $S(G)$ and the normal subgroup of $A(G)$ that is generated by $S(G)$ determine the same $\mathscr{S}(G)$.

Proof. (1) Let $\tau \in A(G)$ and $C \in \mathscr{S}(G)$. If $\sigma \in S(G)$, then $\tau \sigma \tau^{-1} \in S(G)$ and so $C \tau \sigma \tau^{-1}=C$. Therefore $C \tau \sigma=C \tau$ for all $\sigma \in S(G)$ and so $C \tau \in \mathscr{S}(G)$.

(2) If $\sigma \in S(G), \tau \in A(G)$, and $C \in \mathscr{S}(G)$, then $C \tau \sigma \tau^{-1}=C \tau \tau^{-1}=C$ since $C \tau \in \mathscr{S}(G)$ by our hypothesis. Thus each conjugate of an element in $S(G)$ fixes each element of $\mathscr{S}(G)$ and the desired conclusion follows.

REMARKS. (1) For all of our examples $S(G)$ is normal in $A(G)$.

(2) If $S(G)=\{\alpha \in A(G) \mid G(g) \alpha=G(g)$ for all $g \in G\}$, the group of generalized contractors, then $\mathscr{S}(G)=\mathscr{C}(G)$ and each subgroup of $S(G)$ gives the same $\mathscr{S}(G)$.

We conclude this section with a construction that is essentially given in [19] and will yield a dual Galois correspondence between certain characteristic subgroups of an $l$-group $G$ and the normal subgroups of $A(G)$.

If $H$ is a subgroup of $G$, let

$$
H \mu=\{\tau \in A(G) \mid-x+x \tau \in H \text { for all } x \in G\},
$$

and if $K$ is a subgroup of $A(G)$, let

$$
K \nu=[\{-x+x \tau \mid x \in G \text { and } \tau \in K\}],
$$

and let $K \rho$ be the convex $l$-subgroup of $G$ generated by $K \nu$. The verification of the next four propositions are similar to those given in [19] and will be omitted.

2.10. $H \mu$ is a subgroup of $A(G)$.

2.11. $K \rho$ is an l-ideal of $G$.

2.12. If $K$ is a normal subgroup of $A(G)$, then $K \rho$ is a characteristic subgroup of $G$.

2.13. If $H$ is a characteristic subgroup of $G$, then $H \mu$ is a normal subgroup of $A(G)$. 
Note that for any subgroup $K$ of $A(G), K \rho \mu \supseteq K$ and, for any convex $l$-subgroup $H$ of $G, H \mu \rho \subseteq H$. It follows that $K \rho \mu \rho=K \rho$ and $H \mu \rho \mu=H \mu$. Thus we have the following theorem.

THEOREM 2.14. There is a one-to-one correspondence between the characteristic subgroups of $G$ of the form $K \rho$ and the normal subgroups of $A(G)$ of the form $H \mu$.

3. Shifting subgroups. A convex $l$-subgroup $C$ of $G$ is called a shifting subgroup (s-subgroup) if for each $\tau \in A(G)$ either $C \tau=C$ or $C \tau \cap C=0 . G$ is said to be $s$ simple if $G$ and 0 are the only s-subgroups of $G$. $G$ is completely s-reducible if each $s$-subgroup of $G$ is a cardinal summand. Clearly any characteristic subgroup is an $s$-subgroup. In addition, if $C \in \mathscr{C}(G)$ and $C$ is minimal with respect to being a convex $l$-subgroup, $l$-ideal, polar, lex-subgroup (defined in $\$ 4$ ), or cardinal summand, then $C$ is an $s$-subgroup.

We list below several assertions concerning s-subgroups, most of which are easily proven.

3.1. If $C$ is an $s$-subgroup of $G$ and if $\tau \in A(G)$, then $C \tau$ is an $s$-subgroup of $G$.

3.2. If $D$ is an $s$-subgroup of $C$ and $C$ is an $s$-subgroup of $G$, then $D$ is an $s$ subgroup of $G$.

3.3. If $C$ is an $s$-subgroup of $G$, then so is $C^{\prime \prime}$.

3.4. If $G=A \boxplus B$ and $C$ is an $s$-subgroup of $G$, then $C \cap A$ is an $s$-subgroup of $A$. Thus the set $\mathscr{D}(G)$ of all $s$-subgroups of $G$ satisfies (ii) and (iii) of our definition of $\mathscr{S}(G)$.

3.5. The intersection of an arbitrary collection of $s$-subgroups of $G$ is an $s$ subgroup. Thus $\mathscr{D}(G)$ is a complete lattice with respect to inclusion. In general $\mathscr{D}(G)$ is neither modular nor a sublattice of $\mathscr{C}(G)$.

3.6. If $C$ is a nonzero characteristic subgroup of $G$ and if $D$ is an $s$-subgroup of $G$ containing $C$, then $D$ is characteristic. Thus the collection of nonzero characteristic subgroups of $G$ is a dual ideal of the lattice $\mathscr{D}(G)$.

3.7. If $C$ is an $s$-subgroup of $G$, then $\bigvee\{C \tau \mid \tau \in A(G)\}=\sum C \tau_{i}(i \in I)$ where $\left\{\tau_{i} \mid i \in I\right\}$ is a system of representatives of the cosets of that subgroup of $A(G)$ consisting of those $l$-automorphisms that fix $C$.

3.8. If $G$ is simple, then $G$ is $s$-simple. If $G$ is $s$-simple, then $G$ is characteristically simple.

Proof. If $C$ is a nonzero s-subgroup of a simple $l$-group $G$, then $\bigvee\{C \tau \mid \tau \in A(G)\}$ is a characteristic subgroup of $G$. Thus $G=\sum C \tau_{i}$ (as in 3.7) and so $C$ is a cardinal summand of $G$. Therefore $C$ is normal in $G$ and so $C=G$. The second assertion is clear.

3.9. A characteristically simple $o$-group is $s$-simple and conversely.

3.10. If $G$ is completely reducible and $s$-simple, then $G$ is simple.

Proof. Suppose (by way of contradiction) that $A$ is a proper $l$-ideal of $G$ and choose $g \in G \backslash A$. Let $M$ be an $l$-ideal that is maximal with respect to $A \subseteq M$ and $g \notin M$, and let $K$ be the intersection of all $l$-ideals of $G$ that properly contain $M$. 
Now $G=M \boxplus L$ and so $K=M \boxplus(K \cap L)$. Clearly $K \cap L$ is a minimal $l$-ideal of $G$ and hence an $s$-subgroup. From this we conclude that $G$ is simple.

3.11. If $G$ is completely reducible, then $G$ is completely $s$-reducible.

Proof. Let $C$ be a proper $s$-subgroup of $G$. Then $K=\bigvee\{C \tau \mid \tau \in A(G)\}=\sum C \tau_{i}$ is an $l$-ideal of $G$ and hence $G=K \boxplus D$. Thus $C$ is a cardinal summand of $G$.

3.12. A prime $s$-subgroup $C$ is either characteristic or totally ordered. $(M \in \mathscr{C}(G)$ is prime if $a, b \in G^{+} \backslash M$ implies $a \wedge b>0$.)

Proof. If $\tau \in A(G)$ and $C \cap C \tau=0$, then $C \tau \subseteq C^{\prime}$ and so (see [11, Theorem 2.1]) $C^{\prime}$ is totally ordered.

An element $C$ of $\mathscr{C}(G)$ is said to be closed if for each subset $\left\{g_{i} \mid i \in I\right\}$ of $C$ for which $g=\bigvee g_{i}(i \in I)$ exists in $G$, it follows that $g \in C$. In particular, each polar is closed. Also if $D \in \mathscr{C}(G)$, then the intersection of all closed subgroups of $G$ containing $D$ is closed, and is called the closure of $D$.

3.13. The closure of an $s$-subgroup (characteristic subgroup) is an $s$-subgroup (characteristic subgroup).

Proof. Let $C$ be an $s$-subgroup and let $D$ be the closure of $C$. Then $D^{+}=$ $\left\{g \in G \mid g=\bigvee g_{i}\right.$ for some subset $\left\{g_{i} \mid i \in I\right\}$ of $\left.C^{+}\right\}$[7, Lemma 3.2]. Suppose that $\tau \in A(G)$ is such that $D \cap D \tau \neq 0$. If $0<d \in D \cap D \tau$, then there exists $\left\{c_{i} \mid i \in I\right\} \subseteq C^{+}$ and $\left\{d_{j} \mid j \in J\right\} \subseteq C^{+}$such that $d=\bigvee c_{i}(i \in I)$ and $d=\bigvee\left(d_{j} \tau\right)(j \in J)$. Thus $0<d=d \wedge d=\bigvee\left(c_{i} \wedge d_{j} \tau\right)(i \in I, j \in J)$. Clearly then $C \cap C \tau \neq 0$, for otherwise $d=0$. Thus $C=C \tau$ and so $D=D \tau$.

THEOREM 3.14. If $G$ is a characteristically simple l-group, then $G=\sum C_{\lambda}(\lambda \in \Lambda)$ where the $C_{\lambda}$ 's are l-isomorphic, s-simple s-subgroups of $G$. Thus the proper s-subgroups are trivially ordered and consist of $\left\{C_{\lambda} \mid \lambda \in \Lambda\right\}$ if $|\Lambda| \geqq 2$.

Proof. If $G$ is $s$-simple, the theorem holds. Otherwise, let $C$ be a proper $s$ subgroup of $G$. Since $\bigvee\{C \tau \mid \tau \in A(G)\}$ is a characteristic subgroup of $G, G=\sum C \tau_{i}$ where $\left\{\tau_{i} \mid i \in I\right\}$ is a subset of $A(G)$. Suppose (by way of contradiction) that $K$ is a proper $s$-subgroup of $G$ that properly contains $C$. If $i \in I$ and if $C \tau_{i} \cap K \neq 0$, then $K \tau_{i} \cap K \neq 0$ and so $K=K \tau_{i}$, whence $C \tau_{i} \subseteq K$. Now

$$
\begin{aligned}
K & =\sum\left(K \cap C \tau_{i}\right) & & (i \in I) \\
& =\sum\left(K \cap C \tau_{j}\right)=\sum C \tau_{j} & & (j \in J \subseteq I)
\end{aligned}
$$

where $J=\left\{i \in I \mid C \tau_{\mathfrak{i}} \cap K \neq 0\right\}$. Since $C \subset K \subset G$, we have $|J| \geqq 2$ and $J \subset I$. Let $j \in J$ and $i \in I \backslash J$. Then the transposition $(i, j)$ induces an $l$-automorphism $\tau$ of $G$ such that $0 \neq K \cap K \tau \neq K$, contradicting the assumption that $K$ is an $s$-subgroup of $G$.

The above argument shows that the proper $s$-subgroups of $G$ are trivially ordered. Thus, because of 3.2, we have that each proper $s$-subgroup is $s$-simple. All of the conclusions of the theorem then follow.

COROLlARY 3.15. A characteristically simple l-group which is cardinally indecomposable is s-simple.

COROLlARY 3.16. If $G$ is characteristically simple, then $G$ is completely s-reducible. 
COROLlaRY 3.17. If $C$ and $D$ are proper s-subgroups of $G$ with $C \subset D$, then $G$ is not characteristically simple. In fact, $\bigvee(C \tau \mid \tau \in A(G)\}$ is a proper characteristic subgroup of $G$.

COROLlARY 3.18. If $G$ is characteristically simple and contains a nonzero abelian s-subgroup $C$, then $G=\sum C_{\lambda}$, where the $C_{\lambda}$ 's are l-isomorphic, abelian, s-simple l-groups. In particular, $G$ is abelian.

THEOREM 3.19. Suppose the collection $\left\{C_{\lambda} \mid \lambda \in \Lambda\right\}$ of all proper s-subgroups of $G$ is trivially ordered and that $|\Lambda| \geqq 2$. Then either $G$ is characteristically simple or $G=C \boxplus D$, where $C$ and $D$ are s-simple characteristic subgroups of $G$ that are not l-isomorphic, and $|\Lambda|=2$.

Proof. For $\gamma, \lambda \in \Lambda(\gamma \neq \lambda), C_{\gamma} \cap C_{\lambda}=0$. Hence $\bigvee\left\{C_{\lambda} \mid \lambda \in \Lambda\right\}=\sum C_{\lambda}(\lambda \in \Lambda)$. Moreover, $\sum C_{\lambda}$ is a characteristic subgroup of $G$. Since $|\Lambda| \geqq 2$ and $\left\{C_{\lambda} \mid \lambda \in \Lambda\right\}$ is trivially ordered, we have that $G=\sum C_{\lambda}$. Suppose that $C \in\left\{C_{\lambda} \mid \lambda \in \Lambda\right\}$ is not characteristic. Then $G=\sum C \tau_{i}$ where $\left\{\tau_{i} \mid i \in I\right\} \subseteq A(G)$, and it follows that there is a one-to-one correspondence $f$ between $I$ and $\Lambda$ such that $C \tau_{i}=C_{i f}$. Since $C \tau_{i}$ is not characteristic for each $i \in I$, it follows that $G$ is characteristically simple.

If some $C$ in $\left\{C_{\lambda} \mid \lambda \in \Lambda\right\}$ is characteristic, then from the above each $C_{\lambda}(\lambda \in \Lambda)$ is characteristic. Since $\left\{C_{\lambda} \mid \lambda \in \Lambda\right\}$ is trivially ordered, $\left\{C_{\lambda} \mid \lambda \in \Lambda\right\}=\{C, D\}$ where $C$ and $D$ are $s$-simple $l$-groups that are not $l$-isomorphic and $G=C \boxplus D$.

THEOREM 3.20. For each $\lambda$ in an indexing set $\Lambda$, let $G_{\lambda}$ be a completely s-reducible l-group. Then $G=\sum G_{\lambda}(\lambda \in \Lambda)$ is completely s-reducible.

Proof. Let $C$ be a nonzero $s$-subgroup of $G$ and let $\Gamma=\left\{\lambda \in \Lambda \mid G_{\lambda} \cap C \neq 0\right\}$. For each $\gamma \in \Gamma, C \cap G_{\gamma}$ is an $s$-subgroup of $G_{\gamma}$, since each element of $A\left(G_{\gamma}\right)$ can be extended to an element of $A(G)$. Therefore $G_{\gamma}=\left(C \cap G_{\gamma}\right) \boxplus D_{\gamma}$ for some $l$-ideal $D_{\gamma}$ of $G_{\gamma}$. Thus

$$
\begin{aligned}
G & =\sum_{(\lambda \in \Lambda \mid \Gamma)} G_{\lambda} \boxplus \sum_{(\gamma \in \Gamma)} G_{\gamma} \\
& =\sum_{(\lambda \in \Lambda \mid \Gamma)} G_{\lambda} \boxplus \sum_{(\gamma \in \Gamma)}\left(\left(C \cap G_{\gamma}\right) \boxplus D_{\gamma}\right)=\sum_{\lambda \in \Lambda \mid \Gamma} G_{\lambda} \boxplus \sum_{\gamma \in \Gamma} D_{\gamma} \boxplus C .
\end{aligned}
$$

Thus $C$ is a cardinal summand of $G$ and hence $G$ is completely $s$-reducible.

THEOREM 3.21. Let $H$ be a characteristically simple l-group and, for each $\lambda \in \Lambda$, let $H_{\lambda}=H$. Then $G=\sum H_{\lambda}(\lambda \in \Lambda)$ is characteristically simple. If $H$ is s-simple and $G$ is not s-simple, then $\left\{H_{\lambda} \mid \lambda \in \Lambda\right\}$ is the collection of proper s-subgroups of $G$. If $|\Lambda|>1$ and $H$ is simple, then each $H_{\lambda}$ is an s-subgroup of $G$ and hence $G$ is not s-simple.

Proof. If $K$ is a nonzero characteristic subgroup of $G$, then $K \cap H_{\lambda} \neq 0$ for some $\lambda \in \Lambda$. Since $K \cap H_{\lambda}$ is a characteristic subgroup of $H_{\lambda}$, we have that $K \cap H_{\lambda}=H_{\lambda}$. For any $\gamma \in \Lambda$, there exists $\tau \in A(G)$ such that $H_{\lambda} \tau=H_{\gamma}$. Thus $H_{\gamma} \subseteq K$ for all $\gamma \in \Lambda$ and so $G=K$. 
Next suppose that $H$ is $s$-simple and that $C$ is a proper $s$-subgroup of $G$. Then $C \cap H_{\lambda} \neq 0$ for some $\lambda \in \Lambda$ and since $C \cap H_{\lambda}$ is an s-subgroup of $H_{\lambda}$, we have that $C \cap H_{\lambda}=H_{\lambda}$. $C$ can have a nontrivial intersection with only one $H_{\lambda}$, for otherwise there exists an $l$-automorphism $\tau$ of $G$ such that $0 \neq C \tau \cap C \neq C$. Thus $C=H_{\lambda}$. Since each permutation of $\Lambda$ induces an $l$-automorphism of $G$, it follows that $\left\{H_{\lambda} \mid \lambda \in \Lambda\right\}$ is the collection of proper $s$-subgroups of $G$.

If $|\Lambda|>1$ and $H$ is simple, then for each $\tau \in A(G), H_{\lambda} \tau \cap H_{\lambda}$ is an l-ideal of $H_{\lambda}$. Thus $H_{\lambda} \tau \cap H_{\lambda}=0$ or $H_{\lambda} \tau \cap H_{\lambda}=H_{\lambda}$ and so each $H_{\lambda}$ is an $s$-subgroup of $G$.

THEOREM 3.22. An l-group $G$ is completely s-reducible if and only if $G=\sum C_{\lambda}$ $(\lambda \in \Lambda)$, where each $C_{\lambda}$ is an s-simple s-subgroup of $G$.

Proof. If $G=\sum C_{\lambda}(\lambda \in \Lambda)$ where each $C_{\lambda}$ is s-simple, then by Theorem 3.20, $G$ is completely $s$-reducible.

For the converse, we may suppose that $G$ is not $s$-simple; for otherwise the desired conclusion follows easily. We first show that $G$ must contain proper $s$-simple $s$-subgroups. If $G$ is characteristically simple, then, by Theorem $3.14, G$ has proper $s$-simple $s$-subgroups. If $G$ is not characteristically simple, then $G$ contains characteristic subgroups $K$ and $L$ such that $0 \neq K \neq G$ and $L$ covers $K$ in the lattice of characteristic subgroups of $G$. Since $G$ is completely s-reducible, $G=K \boxplus K_{1}$ and so $L=K \boxplus\left(L \cap K_{1}\right)$. Clearly $L \cap K_{1}$ is a proper characteristic subgroup of $G$ and is characteristically simple. If $L \cap K_{1}$ is $s$-simple, we have a proper $s$-simple $s$-subgroup of $G$. If $L \cap K_{1}$ is not $s$-simple, let $S$ be a proper $s$-subgroup of $L \cap K_{1}$. Then, by Theorem 3.14, $S$ is $s$-simple. Clearly $S$ is an $s$-subgroup of $G$.

Now let $\mathscr{M}=\left\{C_{\lambda} \mid \lambda \in \Lambda\right.$ be the collection of all proper $s$-simple $s$-subgroups of $G$. Then, since each $C_{\lambda}$ is a cardinal summand of $G$, it follows that $\mathscr{M}$ is a disjoint collection, that is $C_{\lambda} \cap C_{\gamma}=0$ if $\lambda, \gamma \in \Lambda, \lambda \neq \gamma$. Thus $M=\bigvee \mathscr{M}=\sum C_{\lambda}(\lambda \in \Lambda)$. Clearly $M$ is a characteristic subgroup of $G$, and so $G=M \boxplus M_{1}$ where $M_{1}$ is also characteristic. Since $M_{1}$ is also completely $s$-reducible, $M_{1}$ is either $s$-simple or contains proper $s$-subgroups of $G$. The latter case is contradictory and so $M_{1}$ is $s$-simple. If $M_{1} \neq 0$, then $M_{1} \in \mathscr{M}$ and so $M_{1} \subseteq M$, another contradiction. Thus $M_{1}=0$ and $G=M=\sum C_{\lambda}$ where each $C_{\lambda}$ is an $s$-simple $s$-subgroup of $G$.

THEOREM 3.23. For an l-group $G$, the following are equivalent:

(a) $G$ is completely s-reducible.

(b) $G=\sum C_{\lambda}(\lambda \in \Lambda)$, where each $C_{\lambda}$ is an s-simple s-subgroup of $G$.

(c) Each characteristic subgroup is a cardinal summand.

(d) $\mathscr{D}(G)$ is a complemented lattice.

(e) $G=\sum K_{\delta}$, where $\left\{K_{\delta} \mid \delta \in \Delta\right\}$ is the set of minimal characteristic subgroups of $G$.

(f) $G$ is a cardinal sum of characteristically simple l-groups.

(g) $G$ is the join of minimal characteristic subgroups.

(h) $\mathscr{K}(G)$ is a complete, atomic, Boolean algebra.

(i) $\mathscr{K}(G)$ is a Boolean algebra. 
Proof. (a) and (b) are equivalent by Theorem 3.22, and (c), (e), (f), (g), (h), and (i) are equivalent by Theorem 2.2 .

(b) implies (c). Let $0 \neq K$ be a characteristic subgroup of $G$. Then $K \cap C_{\lambda}$ is an $s$-subgroup of the $s$-simple $s$-subgroup $C_{\lambda}$ for each $\lambda \in \Lambda$, and it follows that $K$ is a cardinal summand of $G$.

(c) implies (d). Let $C$ be a proper $s$-subgroup of $G$ and let $K=\bigvee\{C \tau \mid \tau \in A(G)\}=$ $\sum C \tau_{i}$. Then $K$ is characteristic and hence $G=K \boxplus D$, where $D$ is also characteristic. If $D \neq 0$, then $D$ is a complement of $C$ in $\mathscr{D}(G)$. If $D=0$, then $|I|>1$, as $C$ is a proper $s$-subgroup. In this case, any $C \tau_{i} \neq C$ is a complement of $C$ in $\mathscr{D}(G)$.

(d) implies (a). Let $C$ be a proper $s$-subgroup of $G$. Let $K=\bigvee\{C \tau \mid \tau \in A(G)\}=$ $\sum C \tau_{i}$. Since $K \in \mathscr{K}(G) \subseteq \mathscr{D}(G)$, there exists a complement $D$ of $C$ in $\mathscr{D}(G)$. Let $L=\bigvee\{D \tau \mid \tau \in A(G)\}$. Since $K$ is characteristic and $K \cap D=0$, we have that $K \cap L=0$. Both $K$ and $L$ are characteristic and hence so is $K \boxplus L$. It follows that $K \boxplus L$ is the join of $K$ and $L$ in $\mathscr{D}(G)$ and hence $G=K \boxplus L$. Thus $C$ is a cardinal summand of $G$.

COROLlARY 3.24. Each s-subgroup of a completely s-reducible l-group $G$ is either characteristic or an atom in $\mathscr{D}(G)$.

Proof. $G=\sum C_{\lambda}(\lambda \in \Lambda)$ where each $C_{\lambda}$ is an $s$-simple $s$-subgroup of $G$. Suppose that $S$ is a nonzero $s$-subgroup of $G$ and that $S \neq C_{\lambda}$ for any $\lambda \in \Lambda$. Then $S=$ $\sum\left(C_{\lambda} \cap S\right)=\sum C_{\delta}$ where $\delta \in \Delta \subseteq \Lambda$ and $|\Delta| \geqq 2$. If $S$ is not characteristic, then there exists $\tau \in A(G)$ such that $C_{\delta} \tau=C_{\lambda}$ for some $\lambda \in \Lambda \mid \Delta$. Then the transposition $(\delta, \lambda)$ induces an $l$-automorphism $\tau_{1}$ of $G$ such that $0 \neq S \cap S \tau_{1} \neq S$, and this is a contradiction.

COROLlaRY 3.25. Let $G$ be a completely s-reducible l-group, let $\left\{C_{\lambda} \mid \lambda \in \Lambda\right\}$ be the collection of proper s-subgroups of $G$, and suppose that $|\Lambda|>2$. Then $\mathscr{D}(G)$ is a Boolean algebra if and only if $\mathscr{D}(G)=\mathscr{K}(G)$.

Proof. Suppose that $\mathscr{D}(G)$ is a Boolean algebra, let $C \in\left\{C_{\lambda} \mid \lambda \in \Lambda\right\}$, and let $K=\bigvee\{C \tau \mid \tau \in A(G)\}=\sum C \tau_{i}(i \in I)$. Then there exists a unique $D \in \mathscr{D}(G)$ such that $G=K \boxplus D$. If $D \neq 0$, then $C \boxplus D$ is a characteristic subgroup of $G$ that contains $C$. Hence $K \subseteq C \boxplus D$ and so $K=K \cap(C \boxplus D)=(K \cap C) \boxplus(K \cap D)=C$. Thus ${ }^{\circ} C \in \mathscr{K}(G)$. Suppose (by way of contradiction) that $D=0$. Then $K=G$ and $|I|>1$. If $|I|=2$, then since $C$ is an atom in $\mathscr{D}(G)$, we have $|\Lambda|=2$, a contradiction. Thus $|I|>2$. Let $i, j$, and $k$ be distinct elements of $I$. Then $C \tau_{i}$ and $C \tau_{j}$ are distinct complements of $C \tau_{k}$ in $\mathscr{D}(G)$, a contradiction. The converse is immediate from the theorem.

We conclude this section with the remark that the concept of an s-subgroup is lattice theoretic; that is, if $L$ is a lattice with 0 , then an element $x$ of $L$ is a shifting element of $L$ if $x=x \pi$ or $x \wedge x \pi=0$ for each lattice automorphism $\pi$ of $L$. Note that each atom in a lattice is a shifting element. 
4. Characteristically simple l-groups. We derive some results that are, for the most part, corollaries to the theorems in the last two sections. First we supply some needed definitions.

Let $G$ be an $l$-group and let $C \in \mathscr{C}(G)$. $G$ is a lex-extension of $C$ provided that $C$ is prime and $g \in G^{+} \mid C$ implies that $g>C$. If, in addition, $G \neq C$, then $G$ is a proper lex-extension of $C$. $C$ is a lex-subgroup of $G$ if it is a proper lex-extension of some $D \in \mathscr{C}(G)$. If, in addition, $C$ admits no proper lex-extension in $G$, then we say that $C$ is a maximal lex-subgroup of $G$. A lex-subgroup $C$ that is not properly contained in any other lex-subgroup is an $s$-subgroup (see [10, Proposition 3.1]).

A polar $C$ of $G$ is called principal provided that $C=a^{\prime \prime}$ for some $a \in G$. An element $s$ of $G$ is basic if $s>0$ and $\{x \in G \mid 0 \leqq x \leqq s\}$ is totally ordered. It follows that $s^{\prime \prime}$ is a maximal convex $o$-subgroup of $G$ and hence a maximal lex-subgroup. Also $s^{\prime \prime}$ is a minimal polar and each minimal polar is of this form. A subset $S$ of $G$ is a basis for $G$ if $S$ is a maximal set of pairwise disjoint elements and each $s \in S$ is basic.

THEOREM 4.1. For a characteristically simple l-group $G(\neq 0)$, the following are equivalent:

(a) $G$ has a minimal polar.

(b) The collection of lex-subgroups of $G$ contains a maximal element.

(c) $G=\sum C_{i}(i \in I)$ where the $C_{i}$ 's are o-isomorphic characteristically simple o-groups.

(d) $G$ has a basis.

(e) $G$ has a basic element.

(f) Each principal polar is a cardinal summand and $G$ has a closed prime subgroup other than $G$.

Proof. (a) implies (b). Let $P$ be a minimal polar of $G$. By Theorem 3.14, $G=\sum C_{\lambda}$ $(\lambda \in \Lambda)$ where the $C_{\lambda}$ 's are $s$-simple $s$-subgroups of $G$. There exists $\gamma \in \Lambda$ such that $C_{\gamma} \cap P \neq 0$. Since $P$ is an $s$-subgroup of $G$ and $C_{\gamma}$ is $s$-simple, we have $C_{\gamma} \cap P=C_{y}$ and so $C_{\gamma} \subseteq P$. Now $C_{\gamma}$ is a polar and the minimality of $P$ implies that $C_{\gamma}=P$. Thus $C_{y}$ is totally ordered. Since a lex-subgroup is cardinally indecomposable, it follows that $C_{\gamma}$ is maximal in the collection of lex-subgroups of $G$.

(b) implies (c). Let $L$ be maximal in the collection of lex-subgroups. Again $G=\sum C_{\lambda}(\lambda \in \Lambda)$ where the $C_{\lambda}$ 's are $l$-isomorphic $s$-simple $s$-subgroups of $G$. Since $L$ is an s-subgroup, $C_{\gamma} \subseteq L$ for some $\gamma \in \Lambda$; and since $L$ is cardinally indecomposable, $L=C_{\gamma}$. Thus $L$ is $s$-simple and hence the convex $l$-subgroup of $L$ generated by the nonunits of $L$, the lex-kernel of $L$ is trivial [10, Theorem 2.1]. Therefore $L$ is an $o$ group and so condition (c) follows.

(c) implies (d) and (d) implies (e) are trivial.

(e) implies (f). Let $s$ be a basic element of $G$. Again $G=\sum C_{\lambda}(\lambda \in \Lambda)$, where the $C_{\lambda}$ 's are $l$-isomorphic $s$-simple $s$-subgroups of $G$. Clearly there exists $\gamma \in \Lambda$ such that $s \in C_{\gamma}$. It follows that $s^{\prime \prime}=C_{\gamma}$ and so each of the $C_{\lambda}$ 's is totally ordered. It is then 
clear that each principal polar is a cardinal summand and that $G$ contains a closed prime subgroup $M \neq G$.

(f) implies (a). Let $M(M \neq G)$ be a closed prime subgroup of $G$. Since each principal polar is a cardinal summand, $G$ is representable. Let $0<g \in G \backslash M$ and let $C$ be a convex $l$-subgroup of $G$ containing $M$ that is maximal with respect to $g \notin C$. Then $C$ is closed [7, Lemma 3.3] and $C$ contains a unique minimal prime subgroup $N$ and $N=\bigcup b^{\prime}\left(b \in G^{+} \mid C\right)$ [13, Proposition 5.4], and clearly $N=\bigcup b^{\prime}\left(b \in G^{+} \mid C\right.$, $b \leqq g$ ). Let $C^{*}$ be the unique convex $l$-subgroup of $G$ that covers $C$. Since $G$ is representable, $C$ is normal in $C^{*}\left[6\right.$, Corollary 3.2]. Let $b \in G^{+} \mid C, b \leqq g$. Since $C^{*} / C$ is an archimedean $o$-group, there exists an integer $n$ such that $C+g<C+n b$. If $x \in b^{\prime}$, then $x \in(n b)^{\prime}$ and so $x \in(n b \wedge g)^{\prime}$. Since $C$ is prime, $n b \wedge g>0$ and $n b \wedge g \in$ $C+g$. Thus we have $N=\bigcup a^{\prime}(0<a \leqq g$ and $a \in C+g)$. Let $d(d>0)$ be a lower bound for $\{a \mid 0<a \leqq g$ and $a \in C+g\}$ [7, Lemma 3.1]. Then $N=d^{\prime}$ and so $d^{\prime \prime}$ is a minimal polar.

THEOREM 4.2. Let $G$ be a minimal l-ideal of an l-group $H$ and let $C$ be a proper ssubgroup of $G$. Then $G$ is characteristically simple and hence $G=\sum C_{\lambda}(\lambda \in \Lambda)$, where the $C_{\lambda}$ 's are conjugate subgroups of $C$ in $H$.

Proof. $C$ is an $s$-subgroup of $H$. Let $I(H)$ denote the inner automorphism group of $H$. Then $K=\bigvee\{C \sigma \mid \sigma \in I(H)\}$ is a nonzero $l$-ideal of $H$ contained in $G$. Thus $G=K=\sum C \sigma_{i}$ where $\left\{\sigma_{i} \mid i \in I\right\} \subseteq I(G)$.

REMARK. In the above theorem, if $C$ is a minimal $l$-ideal of $G$, then since $G=C \boxplus D$, it follows that $C$ is simple.

THEOREM 4.3. If $G$ is characteristically simple and if $G=A \boxplus B$, where $A$ and $B$ are nonzero and $A$ is cardinally indecomposable, then

(1) $A$ is an s-simple s-subgroup of $G$ and $G=\sum A_{\lambda}(\lambda \in \Lambda)$, where the $A_{\lambda}$ 's are l-isomorphic to $A$.

(2) If $G=C \boxplus D$, then $C=\sum A_{\delta}(\delta \in \Delta)$ for some subset $\Delta$ of $\Lambda$. Hence $C$ is characteristically simple.

Proof. If $\tau \in A(G)$ is such that $A \cap A \tau \neq 0$, then $A=A \cap G=A \cap(A \tau \boxplus B \tau)=$ $(A \cap A \tau) \boxplus(A \cap B \tau)=(A \cap A \tau)$, since $A$ is cardinally indecomposable. Therefore $A$ is a proper $s$-subgroup of $G$ and so $G=\sum A_{\lambda}$ where the $A_{\lambda}$ 's are $l$-isomorphic to $A$. By Theorem 3.14, $A$ is $s$-simple.

If $C$ is a cardinal summand of $G$ and if $C \cap A_{\lambda} \neq 0$, then $C \cap A_{\lambda}=A_{\lambda}$, for otherwise $A_{\lambda}$ (and hence $A$ ) would be cardinally decomposable. It follows that $C=\sum A_{\delta}$ $(\delta \in \Delta \subseteq \Lambda$ ). By Theorem 3.21, $C$ is characteristically simple.

A convex $l$-subgroup $M$ of an $l$-group $G$ that is maximal with respect to not containing some $g \in G$ is called a value of $g$ and a regular subgroup of $G$. If $M$ is regular, then there is a unique convex $l$-subgroup $M^{*}$ of $G$ that covers $M$. The pair $\left(M^{*}, M\right)$ is called a covering pair of $G$. The covering pair is said to be normal if $M$ is a normal subgroup of $M^{*}$. In this case $M^{*} / M$ is an archimedean $o$-group. 
THEOREM 4.4. Let $G$ be a characteristically simple l-group such that each covering pair is normal. If $G$ has a maximal prime subgroup, then $G$ is a subdirect product of $a$ direct product of subgroups of the reals.

Proof. Since the intersection of all maximal prime subgroups is characteristic, it follows that this intersection is zero. Since $G$ covers each maximal prime subgroup $M, M$ is normal in $G$. The map

$$
g \rightarrow(\ldots, M+g, \ldots)
$$

is an $l$-isomorphism of $G$ onto a subdirect product of subgroups of the reals.

COROLlARY 4.5. Let $G$ be a representable characteristically simple l-group. If $G$ contains a maximal prime subgroup or a strong unit, then $G$ is a subdirect product of a direct product of subgroups of the reals.

Proof. If $e$ is a strong unit and $M$ is a value of $e$, then $M$ is a maximal prime subgroup. Also for a representable l-group each maximal prime subgroup is normal [6, Corollary 3.2].

An element of an $l$-group $G$ that has exactly one value is said to be special. If $0<g \in G$ has only a finite number of values, then $g$ has a unique representation $g=g_{1}+\cdots+g_{n}$ where the $g_{i}$ 's are disjoint and special [8, Theorem 3.7]. For an element $g$ in $G$ we shall denote by $G(g)$ the convex $l$-subgroup of $G$ generated by $g$. Then $G(g)=\{x \in G|| x|\leqq n| g \mid$ for some $n>0\}$, and is called a principal convex l-subgroup.

Lemma 4.6 (MCAllister). For an l-group $G$, let

$$
F=\bigvee\{G(g) \mid 0<g \in G \text { and } g \text { is finite valued }\}
$$

Then $F$ is a characteristic subgroup of $G$ and also the l-ideal of $G$ generated by all the special elements of $G$. Moreover

$$
F=\bigcup\{G(g) \mid 0<g \in G \text { and } g \text { is finite valued }\} .
$$

Proof. If $\tau \in A(G)$ and $0<g \in G$ is finite valued, then so is $g \tau$. Thus $F$ is characteristic. Also $g=g_{1}+\cdots+g_{n}$, where the $g_{i}$ 's are disjoint and special and so $G(g)=$ $G\left(g_{1}\right) \boxplus \cdots \boxplus G\left(g_{n}\right)$. It follows that $F$ is the $l$-ideal generated by the special elements. It is easy to verify that if $0<g, h \in G$ are finite valued, then so is $g+h$. Thus $F=\bigcup G(g)$.

REMARK. This lemma generalizes to all $g$ with a fixed bound on the cardinality of its values.

THEOREM 4.7. If $G$ is characteristically simple, contains a special element, and a weak unit, then $G$ is a cardinal sum of a finite number of o-isomorphic characteristically simple o-groups and conversely.

Proof. $F$ is a nonzero characteristic subgroup of $G$. Hence $F=G$. If $g$ is a weak unit, then $g \in G(h)$ for some $0<h \in G$, where $h$ is finite valued. Thus $h$ is also a weak 
unit of $G$ and so we may also assume that $g$ is finite valued. Since we have $g^{\prime}=0$, it follows that $g^{\prime \prime}=G$. Now $g=g_{1}+\cdots+g_{n}$ where the $g_{i}$ 's are disjoint and special. Thus $G$ is the lex-sum of the maximal lex-subgroups $g_{1}^{\prime \prime}, \ldots, g_{n}^{\prime \prime}[10$, Corollary II, p. 100]. Therefore $G=A_{1} \boxplus \cdots \boxplus A_{k}$ where each $A_{i}$ is a lex-subgroup of $G$, and this is the unique decomposition of $G$ into cardinally indecomposable summands. It follows from Theorem 4.3 that the $A_{i}$ 's are $l$-isomorphic $s$-simple $s$-subgroups of $G$. In particular, the lex-kernel of $A_{\mathfrak{i}}$ is zero and so $A_{\mathfrak{i}}$ is a characteristically simple o-group.

LEMMA 4.8. An l-group $G$ is characteristically simple if and only if $0<a, b \in G$ implies $b<n\left(a \tau_{1}+\cdots+a \tau_{k}\right)$ for some $\tau_{1}, \ldots, \tau_{k} \in A(G)$ and some positive integer $n$.

Proof. Clearly the condition is sufficient. If $G$ is characteristically simple, then $b$ must belong to the characteristic subgroup $T$ generated by $a$. Now

$$
T=\bigvee\{G(a) \tau \mid \tau \in A(G)\}=\bigvee\{G(a \tau) \mid \tau \in A(G)\}
$$

Thus $b=b_{1}+\cdots+b_{k}$ where $b_{i} \in G\left(a \tau_{i}\right)(i=1,2, \ldots, k)$ and hence $b_{i} \leqq\left|b_{i}\right|<n_{i} a \tau_{i}$ for some $n_{i}>0$. Therefore $b<n_{1} a \tau_{1}+\cdots+n_{k} a \tau_{k}<n_{1}\left(a \tau_{1}+\cdots+a \tau_{k}\right)+\cdots+$ $n_{k}\left(a \tau_{1}+\cdots+a \tau_{k}\right)=\left(n_{1}+\cdots+n_{k}\right)\left(a \tau_{1}+\cdots+a \tau_{k}\right)=n\left(a \tau_{1}+\cdots+a \tau_{k}\right)$.

Let $\Gamma(G)$ denote the partially ordered set (with respect to inclusion) of all regular subgroups of the l-group $G$. Each $\tau \in A(G)$ induces an $o$-automorphism on $\Gamma(G)$. We shall call $G$ finite valued if each element of $G$ has only a finite number of values. The next theorem shows that for a finite valued $l$-group $G$, the action of $A(G)$ on $\Gamma(G)$ determines whether or not $G$ is characteristically simple.

THEOREM 4.9. For a finite valued l-group $G$, the following are equivalent:

(a) $G$ is characteristically simple.

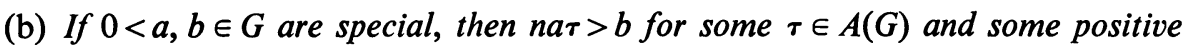
integer $n$.

(c) If $A, B \in \Gamma(G)$, then $A \tau \supseteq B$ for some $\tau \in A(G)$.

Proof. Let $A$ and $B$ be the values of $a$ and $b$ respectively and let $A^{*} \operatorname{cover} A$, and $B^{*}$ cover $B$.

(a) implies (b). By the last lemma, $b<n\left(a \tau_{1}+\cdots+a \tau_{k}\right)$ for some $\tau_{1}, \ldots, \tau_{k} \in$ $A(G)$ and some $n>0$. Thus we may assume that $a \tau_{1} \notin B$. If $a \tau_{1} \notin B^{*}$, then the value $A \tau_{1}$ of $a \tau_{1}$ contains $B^{*}$ and this is the only value of $a \tau_{1}-b$. Moreover, $A \tau_{1}+a \tau_{1}>$ $A \tau_{1}=A \tau_{1}+b$. If $a \tau_{1} \in B^{*}$, then $B+m a \tau_{1}>B+b$ for some positive integer $m$. Thus in either case $m a \tau_{1}>b[8$, p. 114].

(b) implies (c). If $n a \tau>b$, then $n a \tau \notin B$. Thus the value $A \tau$ of $a \tau$ contains $B$.

(c) implies (a). Let $K$ be a proper convex $l$-subgroup of $G$. If $0<g \in G \backslash K$, then $K \subseteq M$, a value of $g$. Let $0<k \in K$ and let $N$ be a value of $k$. Then $K \subseteq M \subseteq N \tau$ for some $\tau \in A(G)$ and $k \tau \notin N \tau$. Thus $K \tau \ddagger K$ and so $K$ is not characteristic.

COROLLARY 4.10. An o-group $G$ is characteristically simple if and only if, for each $A, B \in \Gamma(G)$, there exists $\tau \in A(G)$ such that $A \tau \supseteq B$. 
5. The $l$-group $V\left(\Lambda, R_{\lambda}\right)$. A root system is a partially ordered set $\Lambda$ such that no two incomparable elements have a common lower bound. A root in $\Lambda$ is a maximal chain. Let $\Lambda$ be a root system and for each $\lambda \in \Lambda$, let $R_{\lambda}$ be a subgroup of the reals. Let $\Pi=\Pi \oplus R_{\lambda}(\lambda \in \Lambda)$ denote the direct product of the $R_{\lambda}$ 's and for $v=$ $\left(\ldots, v_{\lambda}, \ldots\right) \in \Pi$, let $S_{v}=\left\{\lambda \in \Lambda \mid v_{\lambda} \neq 0\right\}$. Let $V\left(\Lambda, R_{\lambda}\right)=\left\{v \in \Pi \mid S_{v}\right.$ satisfies the maximum condition $\}$. For $v \in V\left(\Lambda, R_{\lambda}\right)$, let $\Lambda_{v}=\left\{\lambda \in S_{v} \mid v_{\beta}=0\right.$ for all $\left.\beta>\lambda\right\}$. Then $\lambda \in \Lambda_{v}$ is called a maximal component of $v$. An element $v$ in $V\left(\Lambda, R_{\lambda}\right)$ is positive if $v_{\lambda} \geqq 0$ for each $\lambda \in \Lambda_{v}$. With this order $V\left(\Lambda, R_{\lambda}\right)$ is an abelian l-group. If each $R_{\lambda}$ is the group of real numbers, then $V\left(\Lambda, R_{\lambda}\right)$ is a vector lattice. These $l$-groups $V$ are important because each abelian l-group and each (real) vector lattice can be embedded in such a $V$. See [14] for proofs of the above remarks.

THEOREM 5.1 (MCClEARY). The l-group $V=V\left(\Lambda, R_{\lambda}\right)$ is characteristically simple if and only if $V$ is a cardinal sum of a finite number of o-isomorphic characteristically simple o-groups.

Proof. Let $\left\{c_{i} \mid i \in I\right\}$ be a maximal disjoint subset of $V^{+}$. Then $\bigvee\left\{c_{i} \mid i \in I\right\}$ exists and is a unit. Also it is clear that $V$ contains a special element and hence the theorem follows immediately from Theorem 4.7.

REMARK. If each $R_{\lambda}$ equals the reals, then the structure of these characteristically simple 0 -groups is described in the remarks before Corollary 5.9.

COROLLARY 5.2. $V\left(\Lambda, R_{\lambda}\right)$ is s-simple if and only if it is totally ordered and characteristically simple.

Proof. Each totally ordered cardinal summand of an $l$-group is an $s$-subgroup.

An o-automorphism of a partially ordered set $\Delta$ is a permutation $\pi$ of $\Delta$ such that both $\pi$ and $\pi^{-1}$ preserve order.

The following results make it clear that the group of $o$-automorphisms of $\Lambda$ plays an important role in the structure theory of $V\left(\Lambda, R_{\lambda}\right)$.

THEOREM 5.3. For the vector lattice $V=V\left(\Lambda, R_{\lambda}\right)$ the following are equivalent:

(a) Each l-ideal of $V$ is characteristic.

(b) The only o-automorphism of $\Lambda$ is the identity and $\Lambda$ contains only finitely many roots.

Proof. (a) implies (b). Suppose (by way of contradiction) that $\Lambda$ contains infinitely many roots. Then there exists $v \in V$ such that $\left|\Lambda_{v}\right|=\boldsymbol{\aleph}_{0}$ and such that if $\lambda \in \Lambda_{v}$ then $v_{\lambda}=1$. Let $\Lambda_{v}$ be indexed by the natural numbers, say $\Lambda_{v}=\left\{\lambda_{1}, \lambda_{2}, \ldots\right\}$. In $R_{\lambda_{n}}$ consider the $o$-automorphism $x \rightarrow n x$. This induces an $l$-automorphism $\tau$ of $V$ such that for the principal $l$-ideal $V(v)$ we have that $V(v \tau)=V(v) \tau \supset V(v)$, a contradiction. Therefore $\Lambda$ contains only finitely many roots. Next suppose (by way of contradiction) that $\pi$ is an $o$-automorphism of $\Lambda$ such that $\lambda \pi \neq \lambda$ for some $\lambda \in \Lambda$. Without loss of generality, we may suppose that $\lambda \pi\rfloor \lambda$. Let $v \in V$ be such that $v_{\lambda}=1$ and $v_{\beta}=0$ for all $\beta \in \Lambda \mid\{\lambda\}$. If $\tau$ is an $l$-automorphism of $V$ induced by $\pi$, 
then $V(v) \tau \neq V(v)$, a contradiction. Thus the only $o$-automorphism of $\Lambda$ is the identity.

(b) implies (a). Each $l$-ideal of $V$ is the join of subgroups of the form $V(v)$ and so it suffices to show that $V(v) \tau \subseteq V(v)$ for each $\tau \in A(V)$. Since $0<v \in V$ has only a finite number of values, $V(v)=V\left(v_{1}\right) \boxplus \cdots \boxplus V\left(v_{n}\right)$, where each $v_{i}$ is special. Thus, without loss of generality, we may suppose that $v$ is special. Now $\tau$ induces the identity $o$-automorphism on $\Lambda$; hence $v \tau$ has the same value as $v$. Therefore $V(v)=V(v \tau)=V(v) \tau$.

THEOREM 5.4. For the vector lattice $V=V\left(\Lambda, R_{\lambda}\right)$, the following are equivalent:

(a) No nonzero principal l-ideal is characteristic.

(b) For each finite nonvoid trivially ordered subset $X$ of $\Lambda$, there exists an $o$ automorphism $\pi$ of $\Lambda$ such that $X \pi \neq X$.

Proof. (a) implies (b). Let $X=\left\{\lambda_{1}, \ldots, \lambda_{n}\right\}$ be a trivially ordered subset of $\Lambda$ and define $v \in V$ by $v_{\lambda}=1$ if $\lambda \in X$ and $v_{\lambda}=0$ otherwise. By (a) there is an $l$-automorphism $\tau$ of $V$ such that $V(v) \tau \neq V(v)$. Thus $\tau$ induces an $o$-automorphism $\pi$ of $\Lambda$ in which $X \pi \neq X$.

(b) implies (a). If $0<v \in V$ has an infinite number of maximal components, then pick a countable subset of these, say $\left\{\lambda_{1}, \lambda_{2}, \ldots\right\}$, and in $R_{\lambda_{n}}$ consider the $o$ automorphism $x \rightarrow n x$. This induces an $l$-automorphism $\tau$ of $V$ such that $V(v) \tau \neq$ $V(v)$. Suppose that $0<v \in V$ has a finite number of maximal components, $\lambda_{1}, \ldots, \lambda_{n}$. Then there exists an $o$-automorphism $\pi$ of $\Lambda$ so that $\left\{\lambda_{1}, \ldots, \lambda_{n}\right\} \pi \neq\left\{\lambda_{1}, \ldots, \lambda_{n}\right\}$. This induces an $l$-automorphism $\tau$ of $V$ such that $V(v) \tau \neq V(v)$.

Let $\Gamma$ be a root system and let $\Lambda \subseteq \Gamma$. We say that $\Gamma$ is an essential extension of $\Lambda$ if $\Lambda$ is a dual ideal of $\Gamma$ and both $\Gamma$ and $\Lambda$ have the same number of roots.

LEMMA 5.5. If $\Lambda$ is a finite root system, then there exists a finite essential extension $\Gamma$ of $\Lambda$ such that the o-automorphism group $A(\Gamma)$ of $\Gamma$ is trivial.

Proof. We induct on the number $n$ of roots of $\Lambda$. If $n=1$ or if $A(\Lambda)$ is trivial, the theorem holds. Suppose that $n>1$ and pick a root $\Delta_{1}$ of $\Lambda$. Let $\Lambda_{1}=\{\lambda \in \Lambda \mid \lambda$ is an element of some root $\left.\Delta_{i} \neq \Delta_{1}\right\}$. By induction there is an essential extension $\Gamma_{1}$ of $\Lambda_{1}$ such that $A\left(\Gamma_{1}\right)$ is trivial. Adjoin $\Delta_{1} \backslash \Lambda_{1}$ to $\Gamma_{1}$ and add elements to the tail of $\Delta_{1}$ until the resulting root system has a trivial $o$-automorphism group.

COROLlARY 5.6. Each finite-dimensional vector lattice can be embedded in a finite-dimensional vector lattice in which each l-ideal is characteristic.

It is not difficult to show that each totally ordered set can be embedded in one with a trivial $o$-automorphism group. Hence each root system $\Lambda$. with a finite number of roots can be embedded in a root system $\Gamma$ with the same number of roots and such that $A(\Gamma)$ is trivial. Thus each vector lattice $L$ with a finite basis can be embedded in a vector lattice $V$ in which each $l$-ideal is characteristic and such that a basis for $L$ is also a basis for $V$. 
THEOREM 5.7. If $H$ is the divisible hull of a characteristically simple abelian l-group $G$, then $H$ is characteristically simple.

Proof. If $\tau$ is an $l$-automorphism of $G$, then there exists a unique extension of $\tau$ to an $l$-automorphism $\sigma$ of $H$; for if $h \in H$, then $n h \in G$ for some positive integer $n$. Define $h \sigma=(n h \tau) / n$. A routine check shows that $\sigma$ is an $l$-automorphism of $H$. If $0<a, b \in H$, then $m a, m b \in G$ for some positive integer $m$. Thus by Lemma 4.8, there exists $\tau_{1}, \ldots, \tau_{k} \in A(G)$ and a positive integer $n$ such that $m b<$ $n\left((m a) \tau_{1}+\cdots+(m a) \tau_{k}\right)=n m\left(a \sigma_{1}+\cdots+a \sigma_{k}\right)$, where $\sigma_{i}$ is the extension of $\tau_{i}$ to $H$ described above. Therefore $b<n\left(a \sigma_{1}+\cdots+a \sigma_{k}\right)$ and so by Lemma $4.8 H$ is characteristically simple.

Notation. Let $\Lambda$ be a root system and let $V=V\left(\Lambda, R_{\lambda}\right)$ where each $R_{\lambda}$ is the group of reals. Let

$$
\Sigma\left(\Lambda, R_{\lambda}\right)=\left\{v \in V \mid S_{v} \text { is finite }\right\}
$$

and

$$
F\left(\Lambda, R_{\lambda}\right)=\left\{v \in V \mid S_{v} \text { is contained in a finite number of roots }\right\}
$$

Note that both $\Sigma\left(\Lambda, R_{\lambda}\right)$ and $F\left(\Lambda, R_{\lambda}\right)$ are finite valued $l$-groups.

THEOREM 5.8. For a root system $\Lambda$, the following are equivalent:

(a) $\Sigma\left(\Lambda, R_{\lambda}\right)$ is characteristically simple.

(b) $F\left(\Lambda, R_{\lambda}\right)$ is characteristically simple.

(c) For $\alpha, \beta \in \Lambda$, there exists an o-automorphism $\pi$ of $\Lambda$ such that $\alpha \pi \geqq \beta$.

Proof. (a) implies (c). Let $\Sigma=\Sigma\left(\Lambda, R_{\lambda}\right)$, let $\alpha, \beta \in \Lambda$, and let $\Sigma_{\gamma}=\left\{v \in \Sigma \mid v_{\lambda}=0\right.$ for all $\lambda \geqq \gamma\}$ where $\gamma \in\{\alpha, \beta\}$. Then $\Sigma_{\alpha}$ and $\Sigma_{\beta}$ are regular subgroups of $\Sigma$. By Theorem 4.9, there exists $\tau \in A(\Sigma)$ such that $\Sigma_{\alpha} \tau \supseteq \Sigma_{\beta}$. Then $\tau$ induces an $o$-automorphism $\pi$ of $\Lambda$ such that $\alpha \pi \geqq \beta$.

(c) implies (a). Let $\Sigma_{\alpha}$ and $\Sigma_{\beta}$ be as above and let $\pi$ be an $o$-automorphism of $\Lambda$ such that $\alpha \pi \geqq \beta$. Then $\pi$ induces an $l$-automorphism $\tau$ of $\Sigma$ such that $\Sigma_{\alpha} \supseteq \supseteq \Sigma_{\beta}$. Thus by Theorem $4.9, \Sigma$ is characteristically simple.

The proof of the equivalence of (b) and (c) is similar to the one just given.

In an $l$-group $G$, two elements $a$ and $b$ in $G^{+}$are called $a$-equivalent if $a<m b$ and $b<n a$ for some positive integers $m$ and $n$. An $l$-group $H$ is said to be an a-extension of $G$ if $G$ is an $l$-subgroup of $H$ and if for each $h \in H^{+}$there exists $g \in G^{+}$such that $g$ and $h$ are $a$-equivalent. $G$ is said to be $a$-closed if it does not admit a proper $a$-extension. An $a$-closed $a$-extension of $G$ is called an $a$-closure of $G$.

If $G$ is an abelian $o$-group, then by Hahn's embedding theorem (see [14] or [15]) we may assume that $G \subseteq V\left(\Lambda, R_{\lambda}\right)$ where $\Lambda$ is totally ordered and each $R_{\lambda}$ is the group of reals, and $V\left(\Lambda, R_{\lambda}\right)$ is the $a$-closure of $G$. If $G$ is a-closed, then $G=V\left(\Lambda, R_{\lambda}\right)$ and hence $G$ is characteristically simple if and only if, given $\alpha, \beta \in \Lambda$, then $\alpha \pi \geqq \beta$ for some o-automorphism $\pi$ of $\Lambda$. 
COROLlaRY 5.9. If an abelian o-group is characteristically simple, then so is its a-closure.

Proof. This follows from the above and Theorem 4.9.

6. The $l$-group $C(X)$. For a topological space $X$, let $C(X)$ denote the vector lattice of all continuous real-valued functions on $X$, with pointwise order and addition. Many authors (see, for example, [3] or [22]) have shown that an archimedean $l$-group with a strong unit is $l$-isomorphic to an $l$-subgroup of $C(X)$ that contains the constant function 1, where $X$ is a Stone space (i.e. $X$ is extremally disconnected, compact, and Hausdorff). A topological space $X$ is said to be completely regular provided it is a Hausdorff space such that whenever $A$ is a closed set and $x \in X \backslash A$, there exists $f \in C(X)$ such that $x f=1$ and $A f=\{0\}$. For each topological space $Y$ there exists a completely regular space $X$ and a continuous map $\pi$ of $Y$ onto $X$ such that $f \rightarrow \pi f$ is a ring isomorphism of $C(X)$ onto $C(Y)[16$, p. 41]. Thus we shall assume throughout this section that $X$ is completely regular.

It is shown in [16, p. 69] that each prime ring ideal of $C(X)$ is a prime subgroup of $C(X)$; thus if $M$ is a maximal ring ideal of $C(X)$, then $C(X) / M$ is an $o$-field. One calls a topological space $X$ real compact if whenever $M$ is a maximal ring ideal of $C(X)$ and $C(X) / M$ is $o$-isomorphic to the field of real numbers, then

$$
M=C_{x}=\{f \in C(X) \mid x f=0\}
$$

for some $x \in X$. An $f$-ring is a lattice-ordered ring in which $a \wedge b=0$ and $c \geqq 0$ implies $c a \wedge b=a c \wedge b=0$.

LEMMA 6.1. If $G$ is an $f$-ring and $M$ is a minimal prime subgroup of $(G,+)$, then $M$ is a ring ideal.

Proof. Let $0<x \in M$ and $0<g \in G$. Since $M$ is a minimal prime subgroup of $(G,+)$, there exists $0<a \in G \backslash M$ such that $a \wedge x=0$. Thus $a \wedge x g=0$ and so $x g \in M$. Similarly $g x \in M$.

THEOREM 6.2. For a topological space $X$ and $C=C(X)$, the following are equivalent:

(a) If $M$ is a maximal group l-ideal of $C$, then $M=C_{x}$ for some $x \in X$.

(b) $X$ is real compact.

Proof. (a) implies (b) is trivial.

(b) implies (a). Let $M$ be a maximal group $l$-ideal and let $N$ be a minimal prime subgroup of $C$ contained in $M$. By the preceding lemma, $N$ is a ring ideal and hence is contained in a maximal ring ideal $J$ of $C$. Thus $N \subseteq J \subseteq M$. If $J \neq M$, then $C / J$ is a nonarchimedean $o$-field with a maximal convex $o$-subgroup $M / J$, but this is impossible.

In [16] the following results are proven:

(a) Each compact space is real compact (p. 71).

(b) Each metrizable space of nonmeasurable cardinals is real compact (p. 232). 
(c) A discrete space is real compact if and only if its cardinal number is nonmeasurable (p. 163).

(d) Each Lindelöf space is real compact (p. 115).

(e) Each subspace of a Euclidean space is real compact (p. 115).

THEOREM 6.3. If the topological space $X$ is real compact and $C=C(X)$, then $A(C)$ is a splitting extension of the group $P(C)$ of polar preserving l-automorphisms of $C$ by the group $H$ of ring l-automorphisms of $C$.

Proof. Let $A=A(C)$ and $P=P(C)$. It is shown in [12] that the group $P$ is $\{\tau \in A \mid$ there exists $g \in C$ with $x g>0$ for all $x \in X$ and $f \tau=f g$ for all $f \in C\}$. Each ring automorphism is an $l$-automorphism [16, p. 13] and $H=\{\tau \in A \mid$ there exists a homeomorphism $\pi$ of $X$ such that $f \tau=\pi f$ for all $f \in C\}$.

If $\tau \in A$, then $\tau$ induces a permutation of the set $\mathscr{M}$ of maximal group $l$-ideals of $C$. Each maximal group $l$-ideal is of the form $C_{x}$ for some $x \in X$. Define $\pi: X \rightarrow X$ by $x \pi=y$, where $C_{x} \tau=C_{y}$. Then $\pi$ is a permutation of $X$. We wish to show that $\pi$ is continuous. Suppose (by way of contradiction) that there exists a net $\left\{x_{\lambda} \mid \lambda \in \Lambda\right\}$ such that $x_{\lambda} \rightarrow x$ and such that $x_{\lambda} \pi$ lies outside of a given $\varepsilon$-neighborhood of $x \pi$ for each $\lambda \in \Lambda$. Pick $f \tau \in C$ such that $\left(x_{\lambda} \pi\right) f \tau=0$ for all $\lambda \in \Lambda$, but $(x \pi) f \tau \neq 0$. Observe that for $y \in X$ and $h \in C$, the following are equivalent:

(i) $y h=0$.

(ii) $h \in C_{y}$.

(iii) $h \tau \in C_{y} \tau=C_{y \pi}$.

(iv) $(y \pi) h \tau=0$.

Thus we have that $x_{\lambda} f=0$ for all $\lambda$, but $x f \neq 0$. This contradicts the assumption that $f$ is continuous. Therefore $\pi$ is a homeomorphism.

Define $\sigma$ from $C$ into $C$ by $(x) f \sigma=(x \pi) f$ for all $x \in X$ and all $f \in C$. Then $\sigma \in H$ and hence $\tau \sigma \in A$. Using the conditions (i) through (iv) above, it is easily shown that $\tau \sigma=\rho \in P$. Therefore $\tau=\rho \sigma^{-1} \in P H$.

Clearly $P \cap H$ is the identity subgroup. Let $\tau \in P$ where $\tau$ is given by $f \tau=f g$ for some $g \in C$ and $\sigma \in H$ where $\sigma$ is given by $f \sigma=\pi f$. Then for $f \in C$ and $x \in X$, $x f \sigma \tau \sigma^{-1}=\left(x \pi^{-1}\right) f \sigma \tau=\left(x \pi^{-1}\right) f \sigma\left(x \pi^{-1}\right) g=(x f)\left(x g \sigma^{-1}\right)$. Since $x g \sigma^{-1}>0$ for all $x \in X$, $\sigma \tau \sigma^{-1} \in P$. Hence $P$ is a normal subgroup of $A$ and so $A$ is a splitting extension of $P$ by $H$.

For $f \in C(X)$ let $K_{f}$ be the closure of the support of $f$, let $C^{*}(X)=\{f \in C(X) \mid f$ is bounded $\}$, and let $C_{K}(X)=\left\{f \in C(X) \mid K_{f}\right.$ is compact $\}$.

COROLlARY 6.4. If $X$ is real compact, then $C_{K}(X)$ is a characteristic subgroup of $C(X)$.

Proof. The following properties of $C_{K}(X)$ are proven in [16]:

(a) $C_{K}(X)$ is an ideal in $C(X)$ (and $\left.C^{*}(X)\right)$ (p. 61).

(b) For any topological space $X, C_{K}(X)$ is the intersection of all free ideals in $C(X)$ or $C^{*}(X)$ (p. 109). If $X$ is real compact then $C_{K}(X)$ is the intersection of all free maximal ideals of $C(X)$ (p. 123). 
We first show that $C_{K}(X)$ is an $l$-ideal of $C(X)$ for any topological space $X$. If $g \in C_{K}(X)$, then $K_{g \vee 0}$ is a closed subset of the compact set $K_{g}$ and hence compact. Thus $g \vee 0 \in C_{K}(X)$. If $h \in C(X)$ is such that $0<h<g \in C_{K}(X)$, then again $K_{h}$ is compact and so $h \in C_{K}(X)$.

Now if $X$ is real compact, then by the theorem, $A=P H$ and clearly $C_{K}(X)$ is mapped to itself under the action of elements from both $P$ and $H$.

THEOREM 6.5. If $X$ is real compact and the group of homeomorphisms of $X$ acts transitively on $X$, then $C_{K}(X)$ is the minimal characteristic subgroup of $C(X)$.

Proof. Let $0<g \in C(X), 0<h \in C_{K}(X)$, let $x \in X$ be such that $x g>0$, and let $y \in K_{h}$. Then there exists a homeomorphism $\pi$ of $X$ such that $y \pi=x$. Now $\pi$ induces an $l$-automorphism $\tau$ of $C(X)$ and $y g \tau=(y \pi) g=x g>0$. It follows by the compactness of $K_{h}$ that there exists $\tau_{1}, \ldots, \tau_{n} \in A(C(X))$ such that $f=g \tau_{1}+\cdots+g \tau_{n}$ and $z f>0$ for all $z \in K_{h}$. Thus a suitable multiple of $f$ exceeds $h$, and so $C_{K}(X)$ is the minimal characteristic subgroup of $C(X)$.

COROLlaRY 6.6. If $X$ is the set of real numbers, then $C_{K}(X)=\{f \in C(X) \mid$ there exists $x, y \in X$ with $x<y$ and $z f=0$ if $z \in X \backslash(x, y)\}$ is the minimal characteristic subgroup of $C(X)$.

COROllary 6.7. If $X$ is discrete with nonmeasurable cardinality, then $\sum R_{x}$ $(x \in X)$, where $R_{x}$ is the reals for each $x \in X$, is the minimal characteristic subgroup of $C(X)=\prod R_{x}(x \in X)$.

Corollary 6.8. If $X$ is the unit circle, then $C_{K}(X)=C(X)$. Hence $C(X)$ is characteristically simple.

COROLlARY 6.9. If $X$ is compact and the group of homeomorphisms acts transitively on $X$, then $C(X)$ is characteristically simple.

Corollary 6.10. Suppose that $X$ is a Stone space such that the group of homeomorphisms acts transitively on $X$. Then $C(X)$ is a complete characteristically simple vector lattice.

As a corollary to the proof of the theorem we have

CoRollary 6.11. If $C=C[0,1]$ and if $K=\{g \in C \mid$ there exists $x, y \in(0,1)$ with $x<y$ such that $z g=0$ if $z \in[0,1] \backslash(x, y)\}$, then $K$ is the minimal characteristic subgroup of $C$.

Using [1, Theorem 11 and Theorem 12], one can construct uncountably many totally ordered compact spaces where each pair of closed intervals are $o$-isomorphic. If the endpoints of such a space are identified, one obtains a compact space $X$ with a transitive group of homeomorphisms. Thus $C(X)$ will be characteristically simple. Note that Corollary 6.8 is a special case of this, where we start with $[0,1]$. 
THEOREM 6.12. If $X$ is compact, then $C(X)$ is characteristically simple if and only if, for each $0<f \in C(X)$ and $x \in X$, there exists $y$ in the support of $f$ and a homeomorphism $\pi$ of $X$ such that $x \pi=y$.

Proof. Suppose that $C=C(X)$ is characteristically simple and let $0<f \in C$. Then the characteristic subgroup $K$ generated by $f$ is $\bigvee C(f) \tau=\bigvee C(f \tau)(\tau \in A(C))$. If there exists $x \in X$ such that $x f \tau=0$ for all $\tau \in A(C)$, then clearly $K \neq C$. Thus given $x \in X$ there exists $\tau \in A(C)$ such that $x f \tau>0$. Now, without loss of generality, $\tau$ is induced by a homeomorphism $\pi$ of $X$ and so $x \pi=y$ for some $y$ in the support of $f$.

Conversely suppose that $0<f$ belongs to a characteristic subgroup $K$ of $C$. For $x \in X$ there exists $y \in X$ and a homeomorphism $\pi$ of $X$ such that $y f>0$ and $x \pi=y$. Without loss of generality $y f>1$ and hence there exists an $l$-automorphism $\tau$ of $C$ such that $f \tau>1$ in some neighborhood of $x$. By the compactness, it follows that the constant function 1 is in $K$ and so $K=C$.

THEOREM 6.13. Suppose that $G$ is an l-subgroup of $C(X)$ containing 1 , where $X$ is a Stone space. Then $G$ is characteristically simple if and only if for each $0<g \in G$ and $x \in X$ there exists an l-automorphism $\tau$ of $G$ such that $x g \tau>0$.

Proof. If $G$ is characteristically simple and if $0<g \in G$ and $x \in X$, then by Lemma $4.8,1<n\left(g \tau_{1}+\cdots+g \tau_{k}\right)$ where $\tau_{1}, \ldots, \tau_{k} \in A(G)$ and $n$ is a positive integer. Thus $x g \tau_{i}>0$ for some $i$.

Conversely let $0<g \in G$ and for each $x \in X$ choose $\tau_{x} \in A(G)$ and a positive integer $n_{x}$ such that $n_{x}(x) \tau_{x}>1$. Let $T_{x}=\left\{y \in X \mid n_{x}(y) g \tau_{x}>1\right\}$. Then the $T_{x}$ 's form an open cover for $X$ and hence there is a finite subcover. Therefore $1<n_{x_{1}} g \tau_{x_{1}}+$ $\cdots+n_{x_{k}} g \tau_{x_{k}}<m\left(g \tau_{x_{1}}+\cdots+g \tau_{x_{k}}\right) \quad$ where $m=\max \left\{n_{x_{1}}, \ldots, n_{x_{k}}\right\}$. Thus the characteristic subgroup of $G$ generated by $g$ contains 1 and hence must be $G$.

We note that in the preceding theorem, we use only the fact that $X$ is compact. Now let $D(X)$ denote the ring of almost finite real-valued continuous functions on the Stone space $X$, and let $P$ be the group of polar preserving $l$-automorphisms of $D(X)$ and $H$ the group of ring $l$-automorphisms of $D(X)$.

THEOREM 6.14. If $X$ is a Stone space, then $A(D(X))$ is a splitting extension of $P$ by $H$.

Proof. Let $D=D(X), A=A(D(X))$ and for $x \in X, D_{x}=\{f \in D \mid x f=0\}$. Let $\tau \in A$ and $f=1 \tau$. We shall show that $f$ has a multiplicative inverse. Suppose (by way of contradiction) that the support $S_{f}$ of $f$ is a proper subset of $X$. Then $X \backslash S_{f}$ is clopen and hence the characteristic function $g$ on $X \backslash S_{f}$ belongs to $D$, and $f \wedge g=0$. Thus $0=1 \wedge g \tau^{-1}$, a contradiction. Define $x g=1 / x f$ for all $x \in X$. Then $g$ is the inverse of $f$.

Define $\sigma$ from $D$ into $D$ by $h \sigma=h f^{-1}$ for all $h \in D$. Then $\sigma \in P$ and $1 \tau \sigma=1$. We now show that $\tau \sigma$ induces a homeomorphism $\pi$ of $X$. Let $x \in X$ and $D_{x} \tau \sigma=M$. Then $M$ is a value of 1 . If $M \subseteq D_{y}$ for some $y \in X$, then $M=D_{y}$ since $1 \notin D_{y}$. If 
$M \nsubseteq D_{y}$ for all $y \in X$, then, by the compactness of $X$, it follows that $1 \in M$, a contradiction. Thus $D_{x} \tau \sigma=D_{y}$ and we define $x \pi=y$. Clearly $\pi$ is a homeomorphism of $X$. (The argument is the same as that given in the proof of Theorem 6.3.) Define $\rho$ by $h \rho=\pi h$. Then $\rho \in H$. Note that the following are equivalent:

(1) $x h=0$.

(2) $h \in D_{x}$.

(3) $h \tau \sigma \in D_{x \pi}$.

(4) $0=(x \pi) h \tau \sigma=x h \rho \tau \sigma$.

Therefore $\rho \tau \sigma$ is a polar preserving $l$-automorphism of $D$ that maps 1 onto 1 and hence it is the identity. Thus $\tau=\rho^{-1} \sigma^{-1} \in P H$. As in Theorem 6.3, $H \cap P$ is the identity subgroup and $P$ is normal in $A$.

THEOREM 6.15. If $X$ is a Stone space that satisfies the condition given in Theorem 6.12 , then $D(X)$ is a characteristically simple, complete, laterally complete vector lattice.

Proof. By the proof of Theorem 6.12, the constant function 1 is in any characteristic subgroup $K$ of $D(X)$. Consider $0<d \in D(X)$ and let $g=1 \vee d$. Then $f \rightarrow f g$ is an $l$-automorphism of the $l$-group $D(X)$ which maps 1 onto $g$. Thus $g \in K$ and hence $d \in K$. Therefore $K=D(X)$.

7. Self-injective $l$-groups. The category of all $l$-groups where the subobjects are $l$-subgroups contains no injectives (see [19]), but as we shall show it does contain self-injectives.

An $l$-group $G$ is said to be self-injective if each $l$-homomorphism of an $l$-ideal $L$ of $G$ can be extended to an $l$-endomorphism of $G$. An $l$-ideal $L$ of $G$ is said to be large in $G$ if whenever $J$ is an $l$-ideal of $G$ such that $J \cap L=0$, then $J=0$.

The proofs of the next three propositions are entirely similar to the corresponding proofs for modules and so we omit them.

7.1. If $G$ is self-injective and $G=A \boxplus B$, then $A$ is self-injective.

7.2. If $L$ is an l-ideal of a self-injective l-group $G$ and $L$ is isomorphic to $G$, then $G=L$ 田 $L^{\prime}$.

7.3. If $G$ is self-injective and $L$ is an l-ideal that is not large in any $l$-subgroup of $G$ except itself, then $G=L \boxplus L^{\prime}$.

An $l$-group $G$ is said to be hyper-archimedean if each $l$-homomorphic image of $G$ is archimedean. It is fairly well known that the following assertions are equivalent (see, for example, [2] or [4]):

(i) $G$ is hyper-archimedean.

(ii) The collection of regular subgroups of $G$ is trivially ordered.

(iii) $G=G(g) \boxplus g^{\prime}$ for each $0<g \in G$.

(iv) If $0<f, g \in G$, then there exists a positive integer $n$ such that

$$
f \wedge n g=f \wedge(n+1) g \text {. }
$$


(v) $G$ is $l$-isomorphic to an $l$-group $H \subseteq \prod R_{\lambda}(\lambda \in \Lambda)$ where each $R_{\lambda}$ is the group of real numbers, and such that if $0<x, y \in H$, then there exists a positive integer $n$ such that $n x_{\lambda}>y_{\lambda}$ for all $\lambda \in \Lambda$ with $x_{\lambda} \neq 0$.

THEOREM 7.4. For a vector lattice $G$, the following are equivalent:

(a) $G$ is self-injective and contains a maximal l-ideal $M$.

(b) $G$ is self-injective and hyper-archimedean.

(c) $G$ is self-injective and archimedean.

(d) $G$ is l-isomorphic to $\sum R_{\lambda}$ where each $R_{\lambda}$ is the group of reals.

Proof. Clearly (b) implies (a) and (b) implies (c).

(a) implies (b). If $G$ is not hyper-archimedean, then there exists a regular subgroup $G_{\alpha}$ such that $G_{\alpha} \subset G^{\alpha} \subset G$ where $G^{\alpha}$ is the $l$-ideal that covers $G_{\alpha}$. If $0<a \in G^{\alpha} \backslash G_{\alpha}$, then $G^{\alpha}=G_{\alpha} \oplus R a$, where $R$ denotes the real numbers. The projection of $G^{\alpha}$ onto $R a$ is an $l$-homomorphism. Now pick $0<g \in G \backslash M$ and $0<b \in G \backslash G^{\alpha}$. Then there exists an $l$-homomorphism $\tau$ of $G^{\alpha}$ onto $R g$ such that $a \tau=g$. Let $\sigma$ be an extension of $\tau$ to an $l$-endomorphism of $G$. Then $G_{\alpha} \subseteq \operatorname{Ker}(\sigma)$. For each positive integer $n, G_{\alpha}+n a<$ $G_{\alpha}+b$, and so $n g=n a \sigma<b \sigma$. Therefore $M<n(M+g)<M+b$ for all $n$, but this contradicts the fact that $G / M$ is an archimedean $o$-group.

(c) implies (b). Again, if $G$ is not hyper-archimedean, then there exists a regular subgroup $G_{\alpha}$ such that $G_{\alpha} \subset G^{\alpha} \subset G$. If $0<b \in G^{\alpha} \backslash G_{\alpha}$, then $G^{\alpha}=G_{\alpha} \oplus R b$ and the projection $\tau$ of $G^{\alpha}$ onto $R b$ is an $l$-homomorphism. Extend $\tau$ to an $l$-endomorphism $\sigma$ of $G$. If $0<a \notin G^{\alpha}$, then $G_{\alpha}+n b<G_{\alpha}+a$ and so $n b=n b \sigma<a \sigma$ for all positive integers $n$, a contradiction.

(d) implies (c). This follows from the fact that each $l$-ideal of $G$ is a cardinal summand.

(b) implies (d). It suffices to prove that $G$ has property $(F)$ (see $\$ 8$ ) or equivalently, that $G(g)$ has a finite basis for each $0<g \in G$. Suppose (by way of contradiction) that $\left\{g_{i} \mid i=1,2, \ldots\right\}$ is an infinite disjoint subset of $G(g)$. Since $0<g_{i} \wedge g \leqq g$ for each $i$, we may assume that $g \geqq g_{i}$ for each $i$. Moreover, we may multiply each $g_{i}$ by a suitable real number to obtain $g_{i} \leqq g$ and $2 g_{i}$ 多 for each $i$. If $x \in \sum G\left(g_{i}\right)$ $(i=1,2, \ldots)$, then $x=x_{1}+x_{2}+\cdots$, where $x_{i} \in G\left(g_{i}\right)(i=1,2, \ldots)$ and all but a finite number of the $x_{i}$ 's are zero. For each $i$, the map $y \rightarrow i y$ is an $l$-automorphism of $G\left(g_{i}\right)$ and this induces an $l$-automorphism $\tau$ of $\sum G\left(g_{i}\right) . \tau$ can be extended to an $l$-endomorphism $\sigma$ of $G$. Note we may assume that $G \subseteq \prod R_{\lambda}(\lambda \in \Lambda)$ and that there exists a positive integer $m$ such that $m g_{\lambda}>(g \sigma)_{\lambda}$ for all $\lambda$ such that $g_{\lambda}>0$, where $g=\left(\ldots, g_{\lambda}, \ldots\right)$. Now $\left(g_{i}\right)_{\lambda}>0$ implies $g_{\lambda} \geqq\left(g_{i}\right)_{\lambda}>0$ and so $m g_{\lambda}>(g \sigma)_{\lambda} \geqq\left(g_{i} \sigma\right)_{\lambda}=$ $\left(i g_{i}\right)_{\lambda}$. Therefore we have that $m g>i g_{i}$ for a fixed integer $m$ and for all $i$. In particular, $m g>2 m g_{2 m}$ which implies that $g>2 g_{2 m}$, a contradiction.

REMARK. A vector lattice $G$ that satisfies (d) of the theorem is characteristically simple. We also note that a completely reducible l-group is self-injective. We have not been able to characterize nonarchimedean self-injective vector lattices. An example of one that is totally ordered is given in $\$ 9$. 
For the remainder of this section, we shall suppose that $G$ is an $l$-group such that $G$ is $l$-isomorphic to $G(g)$ for each $0<g \in G$. We state the following properties, the proofs of which are straightforward.

7.5. If $G$ is archimedean and has a strong unit, then it is $l$-isomorphic to a subdirect sum of reals. (The above hypothesis is not needed for this.)

7.6. If there exists $0<g \in G$ that is finite valued, then $G$ is an $o$-group.

7.7. If $G$ has a nonunit, then $G$ is cardinally decomposable.

THEOREM 7.8. If $G$ is a hyper-archimedean l-group such that $G$ is l-isomorphic to $G(g)$ for each $0<g \in G$, then $G$ is characteristically simple.

Proof. Let $0<a, b \in G$. Now $a=a \wedge b+a_{1}$ and $b=a \wedge b+b_{1}$, where $a_{1} \wedge b_{1}=0$.

Case 1. $a_{1} \neq 0 \neq b_{1}$. Then $G\left(a_{1}\right)$ is $l$-isomorphic to $G\left(b_{1}\right)$ and $G=G\left(a_{1}\right) \boxplus G\left(b_{1}\right) \boxplus$ $D$ for some $l$-ideal $D$ of $G$. There exists an $l$-automorphism $\tau$ of $G$ that interchanges $G\left(a_{1}\right)$ and $G\left(b_{1}\right)$. Since $a \wedge b \in G(a)$ and $G\left(a_{1}\right) \subseteq G(a)$, we have that

$$
b=a \wedge b+b_{1} \in G(a)+G\left(a_{1}\right) \tau \subseteq G(a)+G(a) \tau .
$$

Case 2. $b_{1}=0$. Then $b \leqq a$ and hence $b \in G(a)$.

Case 3. $a_{1}=0$. Then $a \leqq b$ and hence $G(a) \subseteq G(b)$. Therefore $G=G(b) \boxplus b^{\prime}=$ $G(a) \boxplus D \boxplus b^{\prime}$ for some $l$-ideal $D$ of $G$. Thus $b=x+y \in G(a) \boxplus D$. If $y=0$, then $b=x \in G(a)$. If $y \neq 0$, then $y>0$ and hence $G=G(a) \boxplus G(y) \boxplus b^{\prime}$. There is an $l$-automorphism $\tau$ of $G$ interchanging $G(a)$ and $G(y)$. Therefore $b \in G(a) \boxplus G(y)=$ $G(a) \boxplus G(a) \tau$. Thus $G$ is the characteristic subgroup generated by $a$ and so $G$ is characteristically simple.

8. Embedding in characteristically simple $l$-groups. In this section we prove that any $l$-group can be embedded in an algebraically simple l-group. In addition we prove that a representable (abelian) $l$-group can be embedded in a characteristically simple representable (abelian) $l$-group.

THEOREM 8.1. Each l-group can be embedded as an l-subgroup of an algebraically simple l-group.

Proof. By [21] we may assume that $G$ is an $l$-subgroup of $\mathscr{P}(F)$, where $\mathscr{P}(F)$ is the $l$-group of all $o$-permutations of a totally ordered field $F$. Thus it suffices to embed $\mathscr{P}(F)$ in an algebraically simple group. Without loss of generality, $F$ contains the rational field. Now $\mathscr{P}(F)$ is doubly transitive on $F$ for if $c<d \in F$ then the map $y \rightarrow(d-c) y+c$ maps 0 onto $c$ and 1 onto $d$.

Let $M$ be the field of power series in $x$ with coefficients in $F$, lex-ordered so that

$$
\cdots \ll x^{-2} \ll x^{-1} \ll 1 \ll x \ll x^{2} \ll \cdots .
$$

Let $U^{*}=\{m \in M \mid m$ exceeds each positive integer $\}$ and let $\pi$ be a one-to-one map of $U^{*}$ onto a set $U$ such that $U \cap M=\varnothing$. Now $\pi$ induces a total order on $U$ and we shall consider $M \cup U$ as a totally ordered set where $M<U$. 
Next we shall show that any two closed intervals of $M \cup U$ are isomorphic. It suffices to show that $[a, b]$ is $o$-isomorphic to $[c, d]$ where $a, b \in M, a<b$ and $c, d \in M \cup U, c<d$. This is clear if $c, d \in M$ or if $c, d \in U$. Suppose that $c \in M$ and $d \in U$ and let $U^{*}$ be as above. Let $b_{1}>b_{2}>\cdots>b_{\alpha}>\cdots$ be an inversely wellordered coinitial sequence in $U^{*}$. Then $n<b_{\alpha}$ for all integers $n$ and all $\alpha$; and if $n<y$ for all integers $n$, then $b_{\alpha}<y$ for some $b_{\alpha}$. Thus $b_{\alpha} \pi<d$ and $c<x^{n}$ for some $b_{\alpha}$ and some positive integer $n$. Since any two intervals of $M$ are $o$-isomorphic, there exists an $o$-isomorphism $f$ of $\left[c, x^{n}, x^{n+1}, \ldots\right)$ onto $[1,2,3, \ldots)$ and an $o$-isomorphism $g$ of $\left(\ldots, b_{\alpha+1} \pi, b_{\alpha} \pi, d\right]$ onto $\left(\ldots, b_{\alpha+2}, b_{\alpha+1}, b_{\alpha}\right]$. Since $\left[c, x^{n}, x^{n+1}, \ldots\right)$ $\cup\left(\ldots, b_{\alpha+1} \pi, b_{\alpha} \pi, d\right]=[c, d]$ and $[1,2,3, \ldots) \cup\left(\ldots, b_{\alpha+2}, b_{\alpha+1}, b_{\alpha}\right]=\left[1, b_{\alpha}\right]$, $f$ and $g$ induce an $o$-isomorphism of $[c, d]$ onto $\left[1, b_{\alpha}\right]$. Since $\left[1, b_{\alpha}\right]$ is contained in $M,\left[1, b_{\alpha}\right]$ is $o$-isomorphic to $[a, b]$. Therefore $[c, d]$ is $o$-isomorphic to $[a, b]$.

Next we repeat a similar construction on the lower end of $M \cup U$ and get a totally ordered set $N=L \cup M \cup U$ where $L<M<U$ and any two closed intervals of $N$ are $o$-isomorphic.

Each $o$-permutation of $F$ can be extended to an $o$-permutation of $M$, and by [21], $\mathscr{P}(F)$ is $l$-isomorphic to an $l$-subgroup of $\mathscr{P}(M) . \mathscr{P}(M)$ can be considered as an $l$-subgroup of the l-group $\mathscr{B}(N)$ of all $o$-permutations of $N$ having bounded support (that is, all $o$-permutations that are the identity outside of some bounded interval). By [17], $\mathscr{B}(N)$ is algebraically simple. This completes the proof of the theorem.

An $l$-group $G$ satisfies property $(F)$ if each element in $G^{+}$exceeds at most a finite number of disjoint elements. In the next two theorems we use the fact that a minimal prime subgroup of a representable $l$-group is an $l$-ideal [6, Theorem 3.1].

THEOREM 8.2. If $G$ is a representable (abelian) l-group that satisfies property $(F)$, then $G$ is l-isomorphic to an l-subgroup of a representable (abelian) l-group that satisfies property $(F)$ and is characteristically simple.

Proof. Let $\left\{s_{\lambda} \mid \lambda \in \Lambda\right\}$ be a basis for $G$. For each $\lambda \in \Lambda$, let $M_{\lambda}$ be a minimal prime subgroup of $G$ such that $s_{\lambda} \notin M_{\lambda}$. We may assume that $G \subseteq \sum G_{\lambda}(\lambda \in \Lambda)$, where $G_{\lambda}=G / M_{\lambda}$ for each $\lambda \in \Lambda$. Note that each $G_{\lambda}$ is an $o$-group. Define a total order on $\Lambda$ and use this to lexicographically order $\sum \oplus G_{\lambda}(\lambda \in \Lambda)$. Denote this $o$-group by $K$. For each integer $i$, let $K_{i}=K$ and use the natural order of the integers $Z$ to lexicographically order $\sum \oplus K_{i}(i \in Z)$ and call this $o$-group $L$. For each $\lambda \in \Lambda$, let $H_{\lambda}=L$ and let $H=\sum H_{\lambda}(\lambda \in \Lambda)$. Then $H$ is characteristically simple and $G$ is $l$-isomorphic to an $l$-subgroup of $H$.

THEOREM 8.3. Each representable (abelian) l-group $G$ is l-isomorphic to an l-subgroup of a representable (abelian) characteristically simple l-group.

Proof. Let $\left\{M_{\delta} \mid \delta \in \Delta\right\}$ be the collection of minimal prime subgroups of $G$. If $\Delta$ is finite, then Theorem 8.2 applies. Hence we assume that $\Delta$ is infinite. 
Let $\mathscr{D}=\left\{A_{\delta} \mid \delta \in \Delta\right\}$ be a partition of $\Delta$ such that $\left|A_{\delta}\right|=|\Delta|$ for each $\delta \in \Delta$, and let $Z$ denote the set of integers. We define a partial order on $\Delta \times Z$ by setting $\left(\delta_{m}, m\right) \leqq$ $\left(\delta_{n}, n\right)$ if and only if $\delta_{m}=\delta_{n}$ and $m=n$ or $m<n$ and there exists $\delta_{m+1}, \ldots, \delta_{n-1} \in \Delta$ such that $\delta_{m} \in A_{\delta_{m+1}}, \quad \delta_{m+1} \in A_{\delta_{m+2}}, \ldots, \delta_{n-1} \in A_{\delta_{n}}$. For $k \in Z$, we will call $\{(\delta, k) \mid \delta \in \Delta\}$ the kth level of $\Delta \times Z$. By definition, distinct elements on the $k$ th level are incomparable.

Let $\left(\delta_{m}, m\right),\left(\delta_{n}, n\right)$, and $\left(\delta_{p}, p\right)$ be elements of $\Delta \times Z$ such that $\left(\delta_{m}, m\right)<\left(\delta_{n}, n\right)$ and $\left(\delta_{m}, m\right)<\left(\delta_{p}, p\right)$ and suppose that $n \leqq p$. Then there exists $\delta_{m+1}, \ldots, \delta_{n-1}$, $\lambda_{m+1}, \ldots, \lambda_{p-1} \in \Delta$ such that $\delta_{m} \in A_{\delta_{m+1}}, \quad \delta_{m+1} \in A_{\delta_{m+2}}, \ldots, \delta_{n-1} \in A_{\delta_{n}}$ and $\lambda_{m} \in A_{\lambda_{m+1}}, \ldots, \lambda_{p-1} \in A_{\lambda_{n}}$. Since $\mathscr{D}$ is a partition, we have that $\delta_{m+1}=\lambda_{m+1}$, $\delta_{m+2}=\lambda_{m+2}, \ldots, \delta_{n}=\lambda_{n}$. Therefore $\left(\delta_{n}, n\right) \leqq\left(\delta_{p}, p\right)$ and it follows that $\Delta \times Z$ is a root system.

For $(\delta, k) \in \Delta \times Z$, we define the cone beneath $(\delta, k)$ to be $\{(\lambda, m) \mid(\lambda, m) \leqq(\delta, k)\}$ and denote this set by $(\delta, k)_{*}$. It is readily verified for $(\gamma, j),(\delta, k) \in \Delta \times Z$ that $(\gamma, j)_{*}$ is $o$-isomorphic to $(\delta, k)_{*}$.

Let $T$ be a root in $\Delta \times Z$. Then $T=\left\{\left(\delta_{k}, k\right) \mid k \in Z\right\}$ for some subset $\left\{\delta_{k} \mid k \in Z\right\}$ of $\Delta$. Let $\Lambda=\bigcup\left(\delta_{k}, k\right)_{*}(k \in Z)$. We assert that the group $A(\Lambda)$ of $o$-automorphisms of $\Lambda$ acts irreducibly on $\Lambda$, i.e., given $(\gamma, j)$ and $(\lambda, l) \in \Lambda$, there exists $\pi \in A(\Lambda)$ such that $(\gamma, j) \pi \geqq(\lambda, l)$. It suffices to show that given $(\gamma, j) \in \Lambda$ and $\left(\delta_{k}, k\right) \in T$ where $(\gamma, j)<\left(\delta_{k}, k\right)$, there exists $\pi \in A(\Lambda)$ such that $(\gamma, j) \pi=\left(\delta_{k}, k\right)$. To do this it will suffice to find $\pi \in A(\Lambda)$ such that $(\lambda, k-1) \pi=\left(\delta_{k}, k\right)$ where $(\lambda, k-1)$ is the unique element in the $(k-1)$ th level between $(\gamma, j)$ and $\left(\delta_{k}, k\right)$. Since there exists $\rho \in A(\Lambda)$ such that $(\lambda, k-1) \rho=\left(\delta_{k-1}, k-1\right)$, we may further suppose that $(\lambda, k-1)=$ $\left(\delta_{k-1}, k-1\right)$. Let $\pi_{k-1}$ be an $o$-isomorphism mapping $\left(\delta_{k-1}, k-1\right)_{*}$ onto $\left(\delta_{k}, k\right)_{*}$. We will construct an $o$-isomorphism $\pi_{k}$ of $\left(\delta_{k}, k\right)_{*}$ onto $\left(\delta_{k+1}, k+1\right)_{*}$ with the property that $\pi_{k} \mid\left(\delta_{k-1}, k-1\right)_{*}=\pi_{k-1}$. Let $\nu$ be a one-to-one mapping of $A_{\delta_{k}}$ onto $A_{\delta_{k+1}}$ such that $\delta_{k-1} \nu=\delta_{k}$. If $\gamma \in A_{\delta_{k}} \mid\left\{\delta_{k-1}\right\}$ let $\pi_{\gamma}$ be an $o$-isomorphism of $(\gamma, k-1)_{*}$ onto $(\gamma \nu, k)_{*}$ and let $\pi_{\delta_{k}}=\pi_{k-1}$. Let

$$
\pi_{k}=\left(\bigcup\left\{\pi_{\gamma} \mid \gamma \in A_{\delta_{k}}\right\}\right) \cup\left\{\left(\left(\delta_{k}, k\right),\left(\delta_{k+1}, k+1\right)\right)\right\} .
$$

Clearly $\pi_{k}$ has the required properties. By induction we obtain a chain of functions

$$
\pi_{k-1} \subseteq \pi_{k} \subseteq \cdots \subseteq \pi_{k+i} \subseteq \cdots
$$

such that $\pi_{k+i}$ is an $o$-isomorphism of $\left(\delta_{k+i}, k+i\right)_{*}$ onto $\left(\delta_{k+i+1}, k+i+1\right)_{*}$ and such that

$$
\pi_{k+i} \mid\left(\delta_{k+i-1}, k+i-1\right)_{*}=\pi_{k+i-1} \quad(i=0,1,2, \ldots) .
$$

Then $\pi=\bigcup \pi_{j}(j=k-1, k, k+1, \ldots)$ is the required function. Define a total order on the set $\Delta$ and let $H$ be the direct sum of the $o$-groups $G / M_{\delta}(\delta \in \Delta)$. For $h \in H$, we define $h>0$ if $h_{\delta}>0$ where $\delta$ is the largest element in the support of $h$. Then $H$ is an o-group. For each $\lambda \in \Lambda$, let $H_{\lambda}=H$. Then $V\left(\Lambda, H_{\lambda}\right)$ is an l-group 
[14], and clearly this group is representable. Let

$L=\left\{v \in V\left(\Lambda, H_{\lambda}\right) \mid\right.$ there exists $\delta_{1}, \ldots, \delta_{n} \in \Delta$ and

$$
\left.k_{1}, \ldots, k_{n} \in Z \text { such that } S_{v} \subseteq \bigcup\left(A_{\delta_{i}} \times\left\{k_{i}-1\right\}\right)\right\} .
$$

Then $L$ is an $l$-subgroup of $V\left(\Lambda, H_{\lambda}\right)$. Let $J$ be the $l$-subgroup of $L$ consisting of those elements whose support is contained in $A_{\delta_{1}} \times\{0\}$. Then $J$ is $l$-isomorphic to $\prod H_{\delta}(\delta \in \Delta)$ since $\left|A_{\delta_{1}}\right|=|\Delta|$. If $\delta \in \Delta$, let $\tau_{\delta}$ be the injection of $G / M_{\delta}$ into $H_{\delta}$. For $\left(\ldots, x_{\delta}, \ldots\right) \in \Pi G / M_{\delta}$, let $\left(\ldots, x_{\delta}, \ldots\right) \tau=\left(\ldots, x_{\delta} \tau_{\delta}, \ldots\right)$. Then $\tau$ is an $l-$ isomorphism of $\prod G / M_{\delta}$ into $\prod H_{\delta}$. Since $G$ is $l$-isomorphic to an $l$-subgroup of $\prod G / M_{\delta}$, we have an $l$-isomorphism of $G$ into $L$. Since any element of $A(\Lambda)$ induces an $l$-automorphism of $L$ and $A(\Lambda)$ acts irreducibly on $\Lambda$, we have that $L$ is characteristically simple. Finally, if $G$ is abelian, then so is $L$.

\section{Examples and open questions.}

EXAMPLE 9.1. A nonarchimedean o-group $G$ such that

(1) $G$ is s-simple but not simple.

(2) $G$ is self-injective.

Let $G=V\left(\Lambda, R_{\lambda}\right)$ where $\Lambda$ is the set of integers with the natural order and $R_{\lambda}$ is the group of real numbers for each $\lambda \in \Lambda$. Clearly $G$ is characteristically simple and, for $o$-groups, this is equivalent to being $s$-simple.

To prove that $G$ is self-injective, we need only to consider the case where $L$ is a proper $l$-ideal of $G$ and $\varphi$ is a nonzero $l$-homomorphism of $L$ into $G$. Since $L$ is a proper $l$-ideal of $G, L=G(g)$ for some $0<g \in G$. Let $n$ be the maximal component of $g$ in $\Lambda$. Each $a \in G$ has a unique representation of the form $a=b+c$ where $b_{i}=a_{i}$ for $i \leqq n$ and $b_{i}=0$ for $i>n, c_{i}=a_{i}$ for $i>n$ and $c_{i}=0$ for $i \leqq n$. Then $b \in L$. Let $m$ be the maximal component of $g \varphi$ in $\Lambda$. Define $a \psi=b \varphi+c \rho$, where $(c \rho)_{i}=0$ for $i \leqq m$ and $(c \rho)_{i}=c_{n+i-m}$ for $i>m$. A straightforward computation shows that $\psi$ is an $l$-endomorphism of $G$.

Note that the root system $\Lambda$ of this example satisfies condition (c) of Theorem 5.8.

EXAMPLE 9.2. An example of an l-group $G$ such that

(1) $G$ is hyper-archimedean and $G$ is l-isomorphic to $G(g)$ for each $0<g \in G$.

(2) $G$ is s-simple and cardinally decomposable, but not completely reducible.

(3) $G$ is l-isomorphic to each nonzero cardinal summand.

Let $\Lambda$ be the trivially ordered set of positive integers and let $G$ consist of all those functions $v$ in $V\left(\Lambda, R_{\lambda}\right)\left(R_{\lambda}=\right.$ reals) which satisfy $v_{i}$ is an integer for each $i \in \Lambda$ and there exists a positive integer $n=n(v)$ such that $v_{i}=v_{i+n}$ for all $i \in \Lambda$. It is easy to show that $G$ is an $l$-group and $G=G(g) \boxplus g^{\prime}$ for each $0<g \in G$. Thus $G$ is hyperarchimedean. Since the support of each $0<g \in G$ is infinite, it follows that $G$ is $l$-isomorphic to $G(g)$ for each $0<g \in G$. Since $G$ has a strong unit, each cardinal summand has a strong unit and so each cardinal summand is isomorphic to $G$.

Since $G$ is not an $o$-group, there exists $0<a, b \in G$ such that $a \wedge b=0$. Thus $G(a+b)=G(a) \boxplus G(b) . G$ is $l$-isomorphic to $G(a+b)$ and therefore cardinally 
decomposable. This argument is valid for any $l$-group $H$ such that $H$ is $l$-isomorphic to $H(h)$ for each $0<h \in H$ and $H$ is not an $o$-group.

Let $C$ be a proper $l$-ideal of $G$. Then $C$ does not contain a strong unit of $G$. There exists $0<a \in C$ and $0<b \in G \backslash C$ such that $a \wedge b=0$ and $a+b$ is a strong unit. Thus $G=G(a+b)=G(a) \boxplus G(b) . a=a_{1}+a_{2}$ and $b=b_{1}+b_{2}$ where $0<a_{1}, a_{2}, b_{1}, b_{2} \in G$, $a_{1} \wedge a_{2}=0, b_{1} \wedge b_{2}=0$. Since $b \notin C$ we may suppose (without loss of generality) that $C \cap G\left(b_{2}\right) \neq G\left(b_{2}\right)$. Now $G=G\left(a_{1}\right) \boxplus G\left(a_{2}\right) \boxplus G\left(b_{1}\right) \boxplus G\left(b_{2}\right)$, and $G\left(a_{1}\right)$ is $l-$ isomorphic to $G\left(b_{2}\right)$. Any $l$-isomorphism between $G\left(a_{1}\right)$ and $G\left(b_{2}\right)$ induces an $l$-isomorphism $\tau$ of $G$ such that $0 \neq C \cap C \tau \neq C$. Thus $G$ is $s$-simple.

No regular subgroup of $G$ is a cardinal summand and so $G$ is not completely reducible.

EXAMPLE 9.3. An example of a characteristically simple vector lattice that contains a special element but is not finite valued (see Theorem 4.7).

First we construct a root system $\Lambda$ from the root systems $\Lambda_{1}$ and $\Lambda_{2}$ given below.

Let $\Lambda_{1}$ be the root system

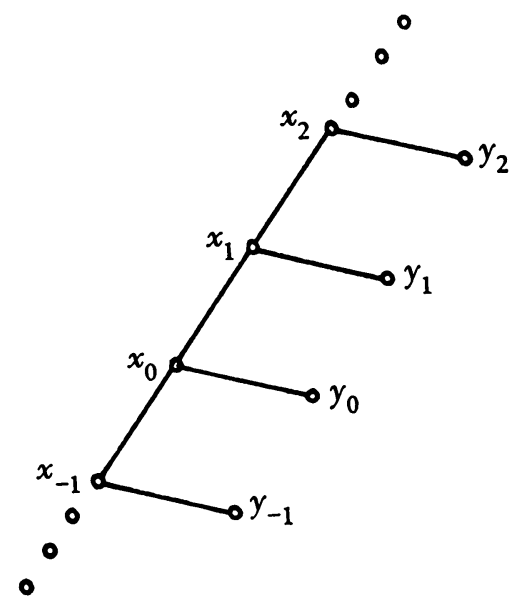

and let $\Lambda_{2}$ be the root system

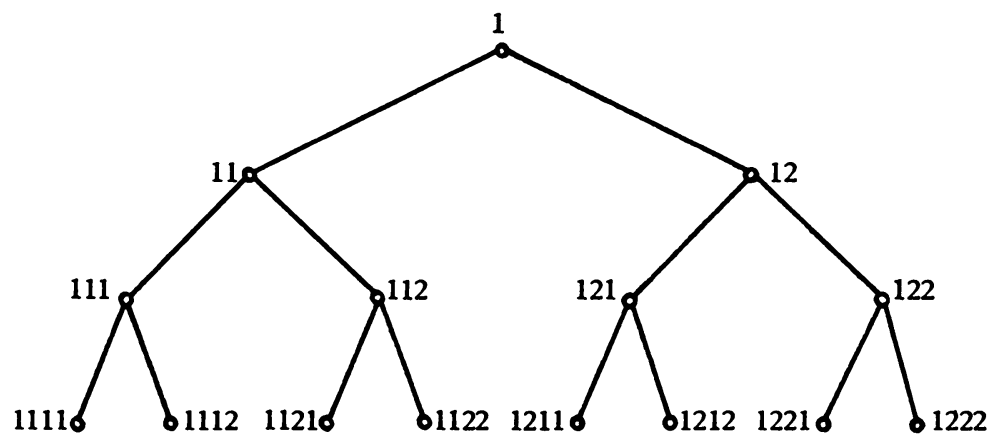

To each $y_{i}$ attach a copy of $\Lambda_{2}$ where we identify the point $y_{i}$ and 1 . Let $\Lambda$ be the resulting root system. 
Note that for each $\alpha$ in $\Lambda$, the set $\{\lambda \in \Lambda \mid \lambda \leqq \alpha\}$ is $o$-isomorphic to $\Lambda_{2}$. Moreover, if $\alpha, \beta \in \Lambda$, then there exists an $o$-automorphism $\pi$ of $\Lambda$ such that $\beta<\alpha \pi$. Let $F$ be the l-ideal of $V\left(\Lambda, R_{\lambda}\right)$ that is defined in Lemma 4.6, where $R_{\lambda}$ is the group of real numbers for each $\lambda \in \Lambda$. Then $F$ has a special element and an element that has an infinite number of values (in fact, infinitely many of both types). To show that $F$ is characteristically simple, it suffices to show that if $0<a, b \in F$ are special, then there exists $\sigma \in A(F)$ such that $a \sigma>b$. Each of $a$ and $b$ has exactly one maximal component in $\Lambda$, say $\alpha$ and $\beta$ respectively. There exists an $o$-automorphism $\pi$ of $\Lambda$ such that $\alpha \pi>\beta$ and $\pi$ induces an $l$-automorphism $\tau$ of $V\left(\Lambda, R_{\lambda}\right)$. If $\sigma=\tau \mid F$, then $a \sigma>b$.

EXAMPLE 9.4. An example of a characteristically simple l-group with a strong unit that is not archimedean.

Let $\mathscr{P}(R)$ denote the l-group of $o$-permutations of the naturally ordered set of real numbers. Let $f \in \mathscr{P}(R)$ be defined by $x f=x+1$ and let $G=\{g \in \mathscr{P}(R) \mid g f=f g\}$. It is known that $G$ is a nonarchimedean simple $l$-group and $f$ is a strong unit in $G$.

EXAMPLE 9.5. An example of an l-group in which no principal convex l-subgroup is characteristic but which has proper characteristic subgroups.

For each natural number $n$, let $R_{n}$ denote the additive group of reals and let $G=\prod R_{n}$. Then no principal convex $l$-subgroup is characteristic, but $\sum R_{n}$ is a characteristic subgroup of $G$.

EXAMPLE 9.6. Examples of characteristic subgroups of an l-group $G$.

(a) The radical, ideal radical, and distributive radical of $G$ (see [7]).

(b) The lex-kernel (i.e., the join of all minimal prime subgroups) of $G$.

(c) The subgroup generated by the singular elements of $G$ ( $s \in G$ is singular if $s>0$ and if $0 \leqq a<s, a \in G$ implies that $a \wedge(s-a)=0)$.

(d) If $S(G)$ is a normal subgroup of $A(G)$, then the $\mathscr{S}$-socle is characteristic.

(e) The $l$-ideal $F$ of Lemma 4.6 .

(f) The subgroup generated by the convex $o$-subgroups of $G$.

(g) The intersection of the maximal $l$-ideals or maximal convex $l$-subgroups of $G$.

(h) The convex $l$-subgroup generated by a characteristic subgroup of the group $G$.

(i) If $A$ is an infinite cardinal and if $X$ is the collection of all $g \in G^{+}$such that the cardinality of any disjoint subset of $G$ bounded by $g$ is less than $A$, then $[X]$ is characteristic.

EXAMPLE 9.7. Examples of characteristically simple l-groups.

(a) The periodic sequences of real numbers.

(b) For each natural number $n$, let $Q_{n}$ denote the additive group of rationals. Then $G=\prod Q_{n} / \sum Q_{n}$ is characteristically simple; in fact $A(G)$ acts transitively on the collection of nonunits of $G$.

(c) $C(X)$ where $X$ is compact with a transitive group of homeomorphisms.

(d) If $\Lambda$ is a root system with property (c) of Theorem 5.8 , then $\Sigma\left(\Lambda, R_{\lambda}\right)$ and $F\left(\Lambda, R_{\lambda}\right)$ are characteristically simple.

(e) If $C$ is a maximal characteristic subgroup of $G$, then $G / C$ is characteristically simple. 
(f) (Bleier) A free abelian l-group of finite rank.

(g) The $l$-group $\mathscr{B}(N)$ of all $o$-permutations of a totally ordered set $N$ having bounded support, where the $o$-permutation group of $N$ acts irreducibly on $N$ [17].

9.8. Let $\mathscr{S}, \mathscr{T}, \mathscr{U}, \mathscr{V}$, and $\mathscr{W}$ denote the classes of simple, $s$-simple, characteristically simple, completely reducible, and completely $s$-reducible $l$-groups respectively. We have noted in 3.8 that $\mathscr{S} \subseteq \mathscr{T} \subseteq \mathscr{U}$, in 3.11 that $\mathscr{V} \subseteq \mathscr{W}$, and it is clear that $\mathscr{S} \subseteq \mathscr{V}$. The $l$-group of Example 9.1 belongs to $\mathscr{T}$ but not $\mathscr{V}$. Let $Q$ and $R$ denote the additive groups of rationals and reals respectively. Then $Q \boxplus R$ belongs to $\mathscr{V}$ and not $\mathscr{U} . R \boxplus R$ belongs to $\mathscr{U} \cap \mathscr{V}$ and not $\mathscr{T}$.

We conclude by asking the following questions.

I. If $G$ is an abelian l-group and if $H$ is its divisible hull, then is $G$ self-injective if and only if $H$ is self-injective?

II. Does Theorem 7.4 hold for abelian l-groups as well as vector lattices?

III. What can be said of s-simple l-groups?

IV. If $G=A \boxplus B$ is characteristically simple, then is $A$ characteristically simple?

V. If $G$ is an archimedean l-group, can $G$ be embedded in a characteristically simple archimedean l-group?

\section{REFERENCES}

1. W. W. Babcock, On linearly ordered topological spaces, Dissertation, Tulane University, New Orleans, La., 1964.

2. K. A. Baker, Topological methods in the algebraic theory of vector lattices, Dissertation, Harvard University, Cambridge, Mass., 1964.

3. S. J. Bernau, Unique representation of Archimedean lattice groups and normal Archimedean lattice rings, Proc. London Math. Soc. (3) 15 (1965), 599-631. MR 32 \#144.

4. A. Bigard, Contribution á la théorie des groupes réticulés, Dissertation, Université de Paris, Paris, France, 1969.

5. G. Birkhoff, Lattice theory, 3rd ed., Amer. Math. Soc. Colloq. Publ., vol. 25, Amer. Math. Soc., Providence, R. I., 1967. MR 37 \#2638.

6. R. D. Byrd, Complete distributivity in lattice-ordered groups, Pacific J. Math. 20 (1967), 423-432. MR 34 \#7680.

7. R. D. Byrd and J. T. Lloyd, Closed subgroups and complete distributivity in lattice-ordered groups, Math. Z. 101 (1967), 123-130. MR 36 \#1371.

8. P. Conrad, The lattice of all convex l-subgroups of a lattice-ordered group, Czechoslovak. Math. J. 15 (90) (1965), 101-123. MR 30 \#3926.

9. - Archimedean extensions of lattice-ordered groups, J. Indian Math. Soc. 30 (1967), 131-160. MR 37 \#118.

10. - Lex-subgroups of lattice-ordered groups, Czechoslovak. Math. J. 18 (93) (1968), 86-103. MR 37 \#1290.

11. - Introduction á la théorie des groupes réticulés, Secrétariat mathématique, Paris, 1967. MR 37 \#1289.

12. P. Conrad and J. Diem, The ring of polar preserving endomorphisms of an abelian latticeordered group, Illinois J. Math. (to appear).

13. P. Conrad and D. McAlister, The completion of a lattice-ordered group, J. Austral. Math. Soc. 9 (1969), 182-208. MR 40 \#2585. 
14. P. Conrad, J. Harvey and C. Holland, The Hahn embedding theorem for abelian latticeordered groups, Trans. Amer. Math. Soc. 108 (1963), 143-169. MR 27 \#1519.

15. L. Fuchs, Partially ordered algebraic systems, Pergamon Press, New York, 1963. MR 30 \#2090.

16. L. Gillman and M. Jerison, Rings of continuous functions, Van Nostrand, Princeton, N. J., 1960. MR 22 \#6994.

17. G. Higman, On infinite simple permutation groups, Publ. Math. Debrecen 3 (1954), 221-226. MR 17, 234.

18. C. Holland, The lattice-ordered group of automorphisms of an ordered set, Michigan Math. J. 10 (1963), 399-408. MR 28 \#1237.

19. W. A. La Bach, An interesting dual Galois correspondence, Amer. Math. Monthly 74 (1967), 991-993. MR 38 \#1036.

20. D. Topping, Some homological pathology in vector lattices, Canad. J. Math. 17 (1965), 411-428. MR $30 \# 4700$.

21. E. C. Weinberg, Embedding in a divisible lattice-ordered group, J. London Math. Soc. 42 (1967), 504-506. MR 36 \#91.

22. B. Z. Vulih, Introduction to theory of partially ordered spaces, Fizmatgiz, Moscow, 1961; English transl., Noordhoff, Groningen, 1967. MR 24 \#A3494; MR 37 \#121.

UNIVERSITY OF HOUSTON, Houston, TEXas 77004

TUlane University, New ORLEans, Louisiana 70118 